\title{
Catching Remote Administration Trojans (RATs)
}

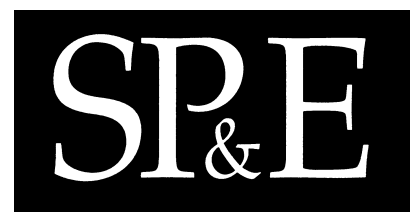

\author{
Zhongqiang Chen ${ }^{1}$ Peter Wei ${ }^{2}$ and Alex Delis ${ }^{3}$ \\ 1 Yahoo! Inc., Santa Clara, CA 95054,E-mail: zqchen@yahoo-inc.com \\ 2 Fortinet Inc., Sunnyvale, CA 94085, E-mail: pwei@fortinet.com \\ ${ }^{3}$ University of Athens, Athens 15771, Greece, E-mail: ad@di.uoa.gr
}

\section{SUMMARY}

A Remote Administration Trojan (RAT) allows an attacker to remotely control a computing system and typically consists of a server invisibly running and listening to specific $T C P / U D P$ ports on a victim machine as well as a client acting as the interface between the server and the attacker. The accuracy of host and/or network-based methods often employed to identify $R A T s$ highly depends on the quality of Trojan signatures derived from static patterns appearing in $R A T$ programs and/or their communications. Attackers may also obfuscate such patterns by having $R A T$ s use dynamic ports, encrypted messages, and even changing Trojan banners. In this paper, we propose a comprehensive framework termed $R A T$ Catcher, that reliably detects and ultimately blocks $R A T$ malicious activities even when Trojans use multiple evasion techniques. Employing network-based methods and functioning in inline mode to inspect passing packets in real time, our RAT Catcher collects and maintains status information for every connection and conducts session correlation to greatly improve detection accuracy. The RAT Catcher re-assembles packets in each data stream and dissects the resulting aggregation according to known Trojan communication protocols, further enhancing its traffic classification. By scanning not only protocol headers but also payloads, $R A T$ Catcher is a truly application layer inspector that performs a range of corrective actions on identified traffic including alerting, packet dropping, and connection termination. We show the effectiveness and efficiency of $R A T$ Catcher with experimentation in both laboratory and real-world settings.

Indexing Terms: Remote Administration Trojans, Trojan detection accuracy, session and event correlation, application layer inspection.

\section{Introduction}

Remote Administration Trojans (RATs) are malicious pieces of code often embedded in legitimate programs through $R A T$-ification procedures [31, 55, 43]. They are stealthily planted and help gain access of victim machines, through patches, games, E-mail attachments, or even in legitimate-looking binaries [31, 6]. Once installed, RATs perform their unexpected or even unauthorized operations [57] and use an array of techniques to hide their traces to remain invisible and stay on victim systems for the long haul. For instance, RAT-ified versions of programs Unix ps and Windows taskmgr.exe keep RATs from appearing in the list of active processes; moreover, by modifying system configurations including the boot-scripts and the Registry database, RAT-binaries often survive system reboots or crashes. A typical RAT consists of a server component running

* Correspondence to: Zhongqiang Chen

Contract/grant sponsor: European Social Funds and National Resources Pythagoras Grant \& Univ. of Athens Research Foundation; contract/grant number: 04-7410 
on a victim machine and a client program acting as the interface between the server and the attacker [28]. The client establishes communications with its corresponding server as soon as the IP address and port of the latter become available through feedback channels such as Email, Instant Messaging and/or Web access [43]. While interacting with a RAT server, an attacker can record keystrokes, intercept passwords, manipulate file systems, and usurp resources of victim systems [43].

By continually changing their name, location, size, and behavior, or employing information encryption, port hopping and message tunneling for its communications, RATs may elude the detection of security protection systems such as firewalls, anti-virus systems (AVs), and intrusion detection/prevention systems (IDSs/IPSs) [42, 16]. Once bound to legitimate programs, RATs in execution inherit a victim's privileges and raise havoc; moreover, they launch attacks against other systems purporting themselves to be superusers [53, 43]. RATs provide the ideal mechanism for propagating malware including viruses, worms, backdoors, and spyware [13, 31, 21, 54]. The number of RATs has been steadily increasing from 1,359 in 2002 to 20,355 in 2004 and their update rates are also impressive; just SubSeven delivered 12 versions in 2002 alone [43]. The number of RATinfected machines is staggering: in 2000, 35\% of security incidents in Korea were Trojaninflicted mostly by Back Orifice (BO) [3] and in 2001, $10 \%$ of intrusions in Israel were due to NetBus and BO [41]. PestPatrol reports that roughly $2 \%$ of all incidents are attributed to RATs [43]. Compromised machines are often used as spring-boards for distributed denial of service attacks, further exacerbating the problem [28].

The best option for avoiding RATs is to verify every piece of software before installation using a-priori known program signatures [19, 7]. This, however, becomes impractical as a comprehensive database of known program signatures is unavailable and RATs are frequently delivered via multiple channels such as patches, attachments, file sharing, or simply Web-site accessing. The polymorphic nature and parasitic mechanisms of RATs render their identification a challenge even if we seek specific and known types of Trojans [11, 12, 2, 43]. Host- and network-based techniques have been widely employed by firewalls, AVs and IDSs/IPSs to detect and block RATs [55, 7]. Static fingerprinting is the predominant method in host-based RAT detection where unique facets of Trojans are extracted to establish a Trojan Database, which entails file names, sizes, locations, checksums, and special patterns in RATs [35, 5, 14, 38]. By periodically scanning every file in a system and matching fingerprints against those in the established database, RATs can be revealed. In addition, monitoring the access of files in the startup folder, registries, autostart files, and configuration scripts of a system is another popular host-based technique that helps identify suspicious activities [40,38]. Network-based methods follow a different philosophy as they examine both the status and activity on TCP/UDP ports to determine any deviation from expected network usage [33, 49]. Abnormal behavior and/or malformed network messages can be detected by monitoring port access patterns and/or analyzing protocol headers of packets exchanged among systems [51]. Similar to host-based methods, unique RAT-manifested telltale patterns in network communications are exploited as signatures to distinguish malicious traffic $[44,51]$. Clearly, the RAT detection accuracy of host- and network-based methods depends on the quality of the Trojan database and signatures used; the latter can be easily obfuscated by attackers using an array of evasion techniques.

In this paper, we propose a comprehensive framework for detecting and dealing with known RATs which employs network-based detection methods and operates in inline mode to inspect and manipulate every passing packet in real-time. Our objective is to enhance the reliability and accuracy of the detection process in comparison with existing anti-Trojan options. To track suspicious RAT activities, our framework monitors network sessions established by both potential Trojans and normal applications, records and maintains state information for their entire lifetime; furthermore, this information is archived even after a session has terminated in order to conduct stateful inspection, intra-session data fusion, and inter-session correlation. By performing packet re-assembly and interpreting the 
resulting aggregations against protocols followed by Trojans, our RAT Catcher morphs data streams into sequences of Trojan messages, facilitating the application-layer inspection and classification of malicious traffic. A number of options are available to manipulate identified $R A T$ sessions ranging from simple alert and log generation to packet dropping and pro-active session blocking/termination. Experimentation with the RAT Catcher shows its effectiveness as well as its efficiency in a range of laboratory and real-world application settings. We organize our paper as follows: Section 2 outlines related work and Section 3 discusses the working mechanisms of RATs. Section 4 presents our proposed framework and Section 5 outlines the findings of our prototyping effort and experimental evaluation. Concluding remarks and future work can be found in Section 6.

\section{Related Work}

Upon activation, a RAT inherits privileges of its program-carrier and complies with the program's expected behavior most of the times, making it challenging to distinguish legitimate activities from malicious ones solely based on program ownership and user profiling $[31,27]$. Verifying that a program is virus-infected is known to be computationally impossible [12]; even searching an executable for known RATs is challenging. The "least privilege principle" is considered an effective way to limit the potential access-scope of Trojanized programs in mulit-user systems [45, 24, 22] even though it is routinely violated [24, 26]. Techniques such as "partitioned protection domains" and "multi-level security models" are also used as means for protection against Trojans [4]; in the former, system partitions provide discretionary access control and in the latter each system entity is statically assigned a security classification [37, 10]. In this context, a Trojan may only obtain information either within the same partition or from entities tagged with lower security classification, thereby limiting its potential damage. Preventing users in different protection domains from sharing programs can work together with integrity models ${ }^{\dagger}$ that compute and store checksums for all files in the systems, and periodically re-calculate these statistics to detect possible file modifications $[35,58]$. Such security procedures often affect the flexibility of a system and are deemed as burden to users.

Fault-tolerance methods have been used to detect unexpected behavior of program segments by treating such deviations as errors [34]. Changes in size, frequency of modification, and ignition rates of programs in conjunction with user profiles $[20,39,56]$ have been used by AVs to detect viruses and RATs [18, 14]. To survive system reboots or crashes, RATs modify system files such as win.ini, system.ini, and/or registry entries in Windows and boot-scripts in Unix systems. To help detect and prevent such systemlevel modifications, a number of host-based security systems allow users to directly enable/disable startup items [38]. Unfortunately, this helps little as it is difficult for users to distinguish legitimate from illegal items and RATs often resort to renaming themselves. Parasitic RATs inject their malicious codes into running processes on-the-fly, effectively shielding themselves from detection [43]. For instance, Trojan Beast inserts itself into active processes such as winlogon and Explorer and becomes a background thread in these programs [47]. Most security systems fail to deal with parasitic RATs as in their effort to curb suspicious activities, they kill legitimate processes as well [47]. A group of RATs can disable firewalls and AVs by killing processes or removing files needed by such security systems [47]. Clearly, the above host-based detection methods may be ineffective as contemporary RATs can readily defy their access control, integrity checking, and behavior profiling. 
RAT servers typically listen on specific ports waiting for instructions from attackers [15]. Utilities such as netstat, Fport and TCPView Pro are designed to monitor active ports for suspicious network activities [15, 33, 49]. A number of firewalls also detect Trojans by searching for applications inducing unauthorized communications. This general approach however may yield false negatives as RATs may bind to legitimate programs and use standard ports [38]. Overall, the effectiveness of techniques based on "static" Trojan characteristics is questioned as soon as RATs commence using non-default ports, hijack ports from other applications, and/or occasionally change communication ports.

By inspecting network traffic and searching for possible Trojan patterns, IDSs/IPSs can establish the intention and/or behavior of data streams [44, 51]. The telltale patterns of Trojans are typically obtained via reverse engineering and data mining techniques [52]. Most IDSs/IPSs heavily base their Trojan-detections on fixed ports and/or simple pattern matching mechanisms, inevitably generating significant false positive or negative rates. Also, this pattern matching is typically conducted only within individual transport-layer $T C P / U D P$ packets rendering IDSs/IPSs vulnerable to evasion attacks [50]. To mitigate evasive attacks, some IDSs/IPSs including Snort and protocol analyzer Ethereal offer traffic stream-reassembly functionality. Unfortunately, this re-assembly feature is only available for pre-specified ports reducing the defense capabilities of such systems significantly [44, 23]. Ethereal is mainly designed as a protocol analyzer that passively collects network traffic without generating any alert for ongoing traffic making it impossible to deliver counter measures in real time [23]. Furthermore, most network-based anti-Trojan systems identify only RAT control channels and do not deal with the content of data channels producing elevated false negative rates [7]. In summary, conventional RATs detection techniques demonstrate limitations and may fail to identify Trojans that resort to a range of advanced evasion techniques.

\section{Characteristics of RATs}

As RATs can essentially capture every screen and keystroke, intruders may obtain account information, passwords, and sensitive computing system data. RATs can also spawn arbitrary numbers of processes on specific $T C P / U D P$ ports, impersonate victims, redirect traffic for specific services to other systems, and launch distributed denial of service $(D D o S)$ attacks. In this section, we examine the salient features of RATs and briefly analyze their capabilities.

\subsection{Frequently Observed Functionalities of RATs}

RATs typically provide attackers with comprehensive command repertoires for file management, process scheduling, and system configuration manipulation. File management features include potentially destructive operations such as delete/move a file or directory on victim systems. The process scheduling component in a RAT permits intruders to create, view, and/or terminate running processes at will. The configuration manipulation element allows RATs to alter the behavior of the victim system by for instance disabling its security features after modifying the Windows Registry. RATs can often operate as device controllers being able to open/close CD-ROMs, disable the mouse and network cards, intercept keystrokes and/or screen snapshots, flip the victim's screen or change its resolution, monitor password dialog boxes and clipboards, capture audio/video of the victim's environment, and finally, crash the victim [43]. The re-direct feature of RATs allows an attacker to chain various services together and ultimately forward the results to a specified destination, making it trivial for intruders to hijack network connections, intercept private data, and inject fake messages. By functioning as packet sniffers, RATs can also monitor a victim's network activities and determine its topology. Furthermore, by scanning the entire system of the victim machine, including its garbage bin, a number of 
RATs can collect personal information such as user accounts, passwords, credit cards, and Email addresses.

Most RATs integrate all the above functionalities and therefore act as a swiss army knife for intruders. In this spirit, Back Orifice (BO), SubSeven, and DeepThroat provide around 60, 100, and 120 commands, respectively. Table I depicts a few commands available in SubSeven and Back Orifice (BO). Here, the SubSeven command IRG can be used to add, remove, or modify system configurations from Registry, command $F F N$ retrieves files from the victim system, and COM can help execute a specific program. Commands $G M I$, $O C D$, and $P W D$ are pertinent to information collection and $T K S \operatorname{logs}$ all keystrokes. Screen snapshots can be captured with $C S S$ and audio/video can be recorded with the help of $R S F$ and IN7. Similar actions are achieved through the repertoire of Back Orifice (BO) as well.

\begin{tabular}{|l|l|l||l|l|l|l|}
\hline$I D$ & command & description & ID & command & description \\
\hline \multicolumn{5}{|c|}{ Some commands provided by SubSeVen } \\
\hline 1 & $G M I$ & get remote machine info & 2 & $P W D$ & get server password \\
\hline 3 & $R A S$ & retrieve RAS passwords & 4 & $G I P$ & get ICQ passwords \\
\hline 5 & $R S H$ & file manager & 6 & $N T F$ & download file \\
\hline 7 & $F F N$ & find files (e.g., wildcard) in given directory & 8 & $T K S$ & key logger \\
\hline 9 & $I N 7$ & 10 & $O C D$ & open CD-ROM \\
\hline 11 & $R W N$ & open web cam & 12 & $F T P$ & start or stop FTP server \\
\hline 13 & $I R G$ & shutdown/restart Windows & 14 & $C O M$ & execute a command \\
\hline \multicolumn{5}{|c|}{ Some commands provided by } & Back Orifice \\
\hline 01 & PING & registry editor & 02 & REBOOT & reboot the remote host \\
\hline 04 & PASSES & ping the current host & 06 & $I N F O$ & Display remote system info \\
\hline 07 & KEYLOG & display remote cached passwords & 09 & DIALOG & display a dialog box \\
\hline $0 \mathrm{E}$ & APPADD & spawn a console application on a TCP port & 14 & HTTPON & enable the HTTP server \\
\hline 19 & PLUGINEXEC & execute a plugin & 20 & $P R O C K I L L$ & kill process given by PROCLIST \\
\hline 23 & REGMAKEKEY & create a key in the registry & 28 & $C A P A V I$ & obtain video stream from device \\
\hline $2 \mathrm{~A}$ & SOUND & play a WAV file & $3 \mathrm{D}$ & MD & make a directory \\
\hline
\end{tabular}

Table I. A few commands provided by SubSeven and Back Orifice (BO)

A number of $R A T$ s offer the proxy functionality that turns a victim machine into a server for services including Telnet, FTP, HTTP, ICQ, and IRC, offering free storage and complete anonymity for attackers. The Trojan Eclypse for instance can be instructed to act as a FTP server; this is depicted by the sample traffic of Table II, which establishes separate channels on different $T C P$ ports for its control and data transmissions much in the spirit of FTP. The apparent protocol similarities in both syntax and semantics between Eclypse and FTP make it difficult to distinguish normal FTP-flows from Trojan-generated traffic. The traffic of Table II shows that the data channel is constructed dynamically (in row 9) once the client submits command NLST to obtain a list of files from the compromised machine (row 8). Clearly, the data channel is server-initiated although the request is clientoriginated and the data port of the client is specified with command PORT dynamically (row 6). Similarly, SubSeven can be configured to act as a proxy server with the help of commands FTP, URL and COM (Table I). RATs can be also used as DDoS attack tools

\begin{tabular}{|c|c|c|c|}
\hline \# & dir & payload & description \\
\hline \multicolumn{4}{|c|}{ protocol: TCP; attacker (denoted as A): 192.168 .5 .143 ; victim (denoted as V): 192.168.5.141 } \\
\hline 1 & $\mathrm{~V}: 3791 \longrightarrow \mathrm{A}: 1074$ & 220 EclYpse 's FTP Server is happy to $\ldots$ & banner in FTP format; telltale: "EclYpse" \\
\hline 2 & $\mathrm{~A}: 1074 \longrightarrow \mathrm{V}: 3791$ & USER (none) & intruder logins with name "(none)" \\
\hline 3 & $\mathrm{~V}: 3791 \longrightarrow \mathrm{A}: 1074$ & 331 Password required for (none). & require password for login \\
\hline 4 & $\mathrm{~A}: 1074 \longrightarrow \mathrm{V}: 3791$ & PASS XXXxxx & password for account name \\
\hline 5 & $\mathrm{~V}: 3791 \longrightarrow \mathrm{A}: 1074$ & 230 User (none) logged in. & attacker logins successfully \\
\hline 6 & $\mathrm{~A}: 1074 \longrightarrow \mathrm{V}: 3791$ & PORT 192,168,5,143,4,51 & specify port for data channel (i.e., $4 * 256+51=1075$ ) \\
\hline 7 & $\mathrm{~V}: 3791 \longrightarrow \mathrm{A}: 1074$ & 200 Port command successful. & server stores data port number \\
\hline 8 & $\mathrm{~A}: 1074 \longrightarrow \mathrm{V}: 3791$ & NLST & request "send host info" \\
\hline 9 & $\mathrm{~V}: 1030 \longrightarrow \mathrm{A}: 1075$ & $(S Y N)$ & establish data channel with port 1075 \\
\hline 10 & $\mathrm{~V}: 3791 \longrightarrow \mathrm{A}: 1074$ & 150 Opening data connection for & data channel has been established \\
\hline 11 & $\mathrm{~A}: 1074 \longrightarrow \mathrm{V}: 3791$ & QUIT & disconnect from the server \\
\hline 12 & $\mathrm{~V}: 3791 \longrightarrow \mathrm{A}: 1074$ & 221 Goodbye. & server closes the session \\
\hline
\end{tabular}

Table II. Traffic generated by the Eclypse (v.1.0) Trojan

provided that enough victims are harvested and instructed to simultaneously flood specific machines. Finally, some RATs can disable, mis-configure, or even kill firewalls, AVs, and IPSs/IDSs incapacitating surveillance. 


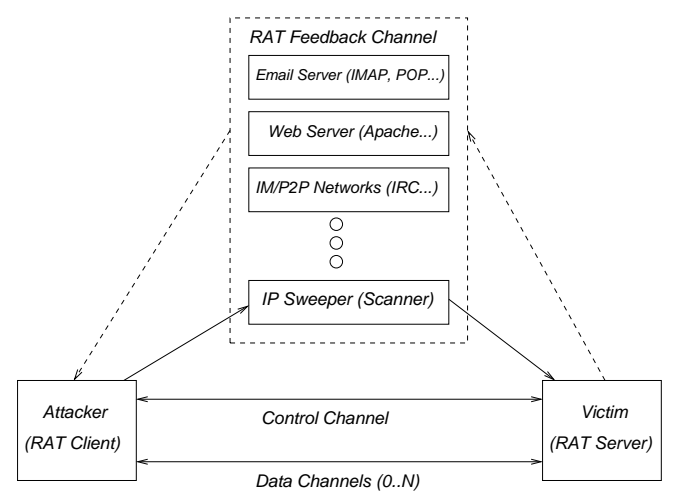

Figure 1. Working mechanisms of a RAT

\subsection{Working Mechanisms of RATs}

Before their installation, $R A T$-servers can be customized via $R A T$-provided configuration packages termed binders. This customization includes the setting of the default TCP/UDP ports utilized by RAT servers, definition of auto-start methods, encryption algorithms and designation of initial login passwords. For instance, EditServer and bo2kcfg are the binders for SubSeven v2.2 and Back Orifice 2000 (BO2K) respectively [43, 42]. Prior to being delivered, $R A T$-servers may be named as software patches or games with the corresponding binders, tricking users into downloading, un-bundling, and finally, executing such malicious programs. Once the servers are configured, they are shipped to victims via a number of delivery channels as described in Figures 1 and 2. During their installation, $R A T$-servers may piggyback themselves to other legitimate programs, termed hosts, so that they are executed every time their hosts are invoked. In this way, a BO2K-server can install itself as a thread to the host program IEXPLORE.EXE [42]. RAT-servers typically run in the background and listen on designated network ports waiting for attacker-issued instructions, leaving victims unaware of their damaging activity.

There is a multitude of avenues to spread Trojans to victim machines as Figure 2 depicts; the most notable for the time being are Instant Messengers (IM) and peerto-peer $(P 2 P)$ systems. With the help of either MSN-messenger or KaZaA, an attacker may freely visit chat-rooms, scan buddy-lists, or even randomly select candidate victims among encountered active users, and subsequently deliver RATs to victims. Additional delivery options include HTTP servers especially created to disseminate Trojans along with regular web-pages, opening Email attachments, execution of malware and distributions for software patches, freewares, and/or games. Hence, anti-Trojan systems are easily defeated if their RAT-detection methods cover only a small portion of such propagation channels.

The IP addresses, TCP/UDP ports, access passwords, and other information of RATservers can be obtained by intruders through feedback channels shown in Figure 1. IM/P2P systems, Email services, and shared folders can even provide auto-notifications between $R A T$-servers and clients. In Guptachar [43] for example, an attacker may set up an $I R C$ server via its IRCBOT ${ }^{\ddagger}$ function by providing a login account nickname; every time a compromised system is activated, it connects to the above $I R C$-server using nickname to upload the victim's $I P$-address and port number. Furthermore, most $R A T$ s resort to multiple methods to outlive system crashes or reboots and evade AVs/IPSs/IDSs [43]. By editing 


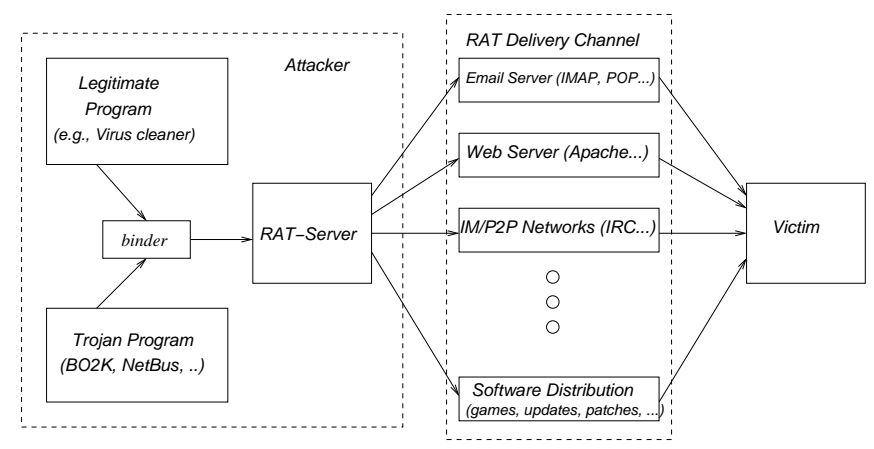

Figure 2. RAT delivery procedure

Registry entries, modifying system files such as win.ini, system.ini and autoexec.bat as well as inserting items on the startup folders, RATs can easily "hide" and be transparently triggered on every reboot. In this regard, host-based detection methods are inferior to their network-based counterparts as far as RAT detection ic concerned.

\subsection{Encrypted RAT Traffic}

RATs such as Back Orifice (BO), BO2K and NetBus v2.0 encrypt their traffic flows to defeat security systems that base their RAT detection on pattern matching techniques. The first half of Table III shows portion of encrypted traffic generated by Back Orifice (BO). The scrambled content of encrypted RAT communications forces many anti-Trojan systems to predominantly use fixed network ports (e.g., the UDP-port for $\mathrm{BO}$ is configurable with default 31337), inevitably leading to false positives/negatives. In $\mathrm{BO}$, the encrypted traffic is generated by applying an XOR operation on the original data and a random stream created with the help of a four-byte encryption key derived from the intruder-specified password ${ }^{\S}$. At the receiver side, the seed (i.e., key) can be guessed via an exhaustive search due to its short range; this seed can then be used to recover the original traffic [42]. Obviously, the $X O R$ operation neither changes the length of the original message nor destroys string patterns; this fact may help identify the BO even if its encrypted traffic is only available.

\begin{tabular}{|c|c|c|c|c|}
\hline \# & dir & size & payload & description \\
\hline \multicolumn{5}{|c|}{ protocol: UDP; attacker (denoted as A): $192.168 .5 .143: 1034$; victim (denoted as V): $192.168 .5 .141: 31337$} \\
\hline \multicolumn{5}{|c|}{ Encrypted messages by Back Orifice } \\
\hline 1 & $\mathrm{~A} \longrightarrow \mathrm{V}$ & 19 & $\mid 9 \mathrm{E}$ F4 C2 EB 8789 A2 04 4E 42 E8 69 3B B9 9855 C3 $8066 \mid$ & encrypted msg \\
\hline 2 & $\mathrm{~V} \longrightarrow \mathrm{A}$ & 37 & |9E F4 C2 EB 8789 A2 047842 E8 69 3A B9 9855 C3 A0 46... & encrypted msg sharing pattern with $\mathrm{msg} 1$ \\
\hline 3 & $\mathrm{~A} \longrightarrow \mathrm{V}$ & 19 & $\mid 9 \mathrm{E}$ F4 C2 EB 8789 A2 04 4E 42 E8 6938 B9 9855 E2 $8066 \mid$ & encrypted msg very similar to $\mathrm{msg} 1$ \\
\hline 4 & $\mathrm{~V} \longrightarrow \mathrm{A}$ & 41 & $\mid 9 \mathrm{E}$ F4 C2 EB 8789 A2 047442 E8 69 3B B9 985562 A0 46... & encrypted $\mathrm{msg}$ very similar to $\mathrm{msg} 2$ \\
\hline 5 & $\mathrm{~A} \longrightarrow \mathrm{V}$ & 19 & |9E F4 C2 EB 8789 A2 04 4E 42 E8 6939 B9 9855 C4 8066 & encrypted $\mathrm{msg}$ very similar to $\mathrm{msg} 1$ \\
\hline 6 & $\mathrm{~V} \longrightarrow \mathrm{A}$ & 49 & |9E F4 C2 EB 8789 A2 04 6C 42 E8 6937 B9 985544 D3 1F... & encrypted $\mathrm{msg}$ very similar to $\mathrm{msg} 2$ \\
\hline \multicolumn{5}{|c|}{ Decrypted Back Orifice traffic } \\
\hline 1 & $\mathrm{~A} \longrightarrow \mathrm{V}$ & 19 & $|2 \mathrm{~A} 212 \mathrm{~A} 515754593 \mathrm{~F} 1300000001000000010000|$ & msg PING starts with string “**!*QWTY?" \\
\hline 2 & $\mathrm{~V} \longrightarrow \mathrm{A}$ & 37 & 2A 21 2A 515754 59 3F 25000000 $000000000120202150 \ldots$ & reply to "PING" (i.e., "PONG"); \\
\hline 3 & $\mathrm{~A} \longrightarrow \mathrm{V}$ & 19 & $|2 \mathrm{~A} 212 \mathrm{~A} 515754593 \mathrm{~F} 1300000002000000200000|$ & cmd PROCESSLIST $(0 \times 20)$ from client \\
\hline 4 & $\mathrm{~V} \longrightarrow \mathrm{A}$ & 41 & |2A 21 2A 51 57 54 593F 29000000 $01000000 \mathrm{~A} 02020 \ldots$ & reply to "PROCESSLIST" (partial) \\
\hline 5 & $\mathrm{~A} \longrightarrow \mathrm{V}$ & 19 & $|2 \mathrm{~A} 212 \mathrm{~A} 515754593 \mathrm{~F} 1300000003000000060000|$ & cmd INFO (0x06) from client \\
\hline 6 & $\mathrm{~V} \longrightarrow \mathrm{A}$ & 49 & |2A 21 2A 51 575459 3F 31000000 0D $000000865379 \ldots \mid$ & reply to "INFO" \\
\hline
\end{tabular}

Table III. Back Orifice generated traffic in both encrypted and decrypted formats 
Through exhaustive search, we can recover the seed 2160 used in the BO-server/client traffic of Table III. The decrypted traffic demonstrates that each BO message commences with the 8-byte magic-string of $* ! * Q W T Y$ ? followed by the 4-byte packet length, 4-byte packet ID, 1-byte message type, the variable length message data and the 2-byte checksum field. The packet length specifies the size of the entire message; for example, the first client message (row 1) is 19-bytes in length. Initially, the BO-client probes the server with command PING (0x01 in the 1-byte message type at index 16) to check the availability of the server. The active server responds with command $P O N G$ along with its version number in row 2. In rows 3 and 5, the client requests information from the victim (i.e., the BO server) with commands PROCESSLIST (0x20) and INFO (0x06). Table III shows that decrypted BO traffic possesses strong message structure and clear semantics. This structure in conjunction with the commands of Table I help attain improved traffic identification based on decrypted content, which is the main techniques used in our RAT Catcher to detect $B O$; a similar approach was also employed by Snort.

The $X O R$-based encryption algorithm used by the international version of $\mathrm{BO} 2 \mathrm{~K}$ can be also reverse-engineered and the original traffic can be recovered, making BO2K detection feasible. Nevertheless, the use of plugin modules in BO2K complicates matters. To this end, the plugins enc_aes, enc_cast and enc_idea encrypt BO2K traffic with the AES, CAST, and IDEA encryption algorithms respectively [46]. It has been shown that such algorithms are resistant to sophisticated differential and linear cryptanalysis techniques [1,30]. Hence, it is impossible to recover original form from encrypted traffic without the requisite encryption keys. For this type of Trojans, the only pragmatic approach for detection has to be based on the RAT external behavior which involves monitoring of message sizes, handshake procedure, and traffic correlation between the two traffic streams within each RAT session.

\begin{tabular}{|c|c|c|c|}
\hline \# & dir & payload & description \\
\hline \multicolumn{4}{|c|}{ protocol: TCP; attacker (denoted as A): 192.168.5.143:1026; victim (denoted as V): $192.168 .5 .141: 20034$} \\
\hline 1 & $\mathrm{~A} \longrightarrow \mathrm{V}$ & $B N \mid 2000020008080500410 \mathrm{C} 69$ 1F 5D 28 5B BC ... & msg starts with $B N$; size: $0 \times 20$; ver: $0 \times 02 ;$ cmd: $0 \times 05$; \\
\hline 2 & $\mathrm{~V} \longrightarrow \mathrm{A}$ & 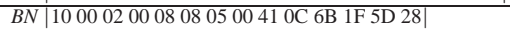 & reply msg; size: 0 x 10 ; ver: $0 \times 02 ;$ cmd: $0 \times 05$; \\
\hline 3 & $\mathrm{~A} \longrightarrow \mathrm{V}$ & $B N|0 \mathrm{~B} 000200 \mathrm{DC} 33300041|$ & client msg; size: $0 \times 0 B ; c m d: 0 \times 30$ \\
\hline 4 & $\mathrm{~V} \rightarrow \mathrm{A}$ & $\overline{\mathrm{DC}} 333000410 \mathrm{C} \mid$ & server msg; size: $0 x 0 \mathrm{C} ; \mathrm{cmd}: 0 \times 30$ \\
\hline 5 & $\overrightarrow{\mathrm{V} \longrightarrow \mathrm{A}}$ & \begin{tabular}{l|l|}
$B N$ & $34000200 \mathrm{~F} 0 \mathrm{AB} 3000410 \mathrm{D} 3 \mathrm{E} F 456 \mathrm{DC} \ldots \mid$ \\
$B N$ & $180002006 \mathrm{~B} 293000410 \mathrm{D} \ldots \mid$ \\
$B N$ & $2 \mathrm{~F} 0002008 \mathrm{~B} 793000410 \mathrm{D} \ldots . \mid$
\end{tabular} & $\begin{array}{l}\text { multiple messages packed into one TCP packet; } \\
\text { msg } 1 \text { is cmd } 0 \times 30 \text { with size } 0 \times 34 \text {; } \\
\text { msg } 2 \text { is } 0 \times 30 \text { with size } 0 \times 18 \ldots\end{array}$ \\
\hline 6 & $\mathrm{~A} \longrightarrow \mathrm{V}$ & \begin{tabular}{l|l|l|}
$B N$ & $1 \mathrm{E} 000200 \mathrm{~F} 0 \mathrm{AB} 500041 \mathrm{C} 957 \ldots \mid$ \\
\end{tabular} & client msg; size: 0x1E; cmd: 0x50 \\
\hline 7 & $\mathrm{~V} \longrightarrow \mathrm{A}$ & 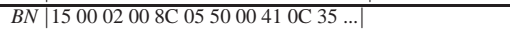 & msg size: $0 \times 15$; ver: $0 \times 02 ; \mathrm{cmd}: 0 \times 50$ \\
\hline
\end{tabular}

Table IV. Portion of NetBus Pro (v.2.0)-generated traffic

NetBus Pro [29] is a noteworthy Trojan in that it uses proprietary encryption algorithms but still offers opportunities for detection based on traffic correlation; it listens to default but reconfigurable TCP-port 20034 and supports plugins enabling the integration of new functionalities [36]. Each NetBus Pro message has a fixed-length header (i.e., 10 bytes) consisting of string " $B N$ " followed by four 2-byte fields namely, message-size, versionnumber (typical value 0x0002), unknown (often a random number), and command-code fields. The variable-sized data section follows the header and its size is specified in the message-size field of the header. Table IV shows a portion of NetBus Pro(v2.0)-generated traffic. Apparently, the beginning of every message is readily determined by string " $B N$ "; correspondingly, the end of the message can be resolved from the message-size field of its header. Multiple messages may be packed in a single TCP packet as is the case with row 5, each of which can be identified through payload content inspection and message structure analysis. Stream-based inspection can also correctly interpret NetBus Pro messages spanning multiple TCP packets. Also, inspection on both streams of a session to ensure their conformance with NetBus Pro protocol specification further improves the detection accuracy. Despite the fact that decryption of RAT traffic is not always feasible, techniques derived from dissecting protocol syntax of RATs combined with analysis of patterns found in message exchanges can be exploited to detect Trojans. The NetBus Pro protocol analyzer outlined by Algorithm 4 of Section 4 functions on this premise. 


\subsection{Diversified Use of Protocols by RATs}

By and large, the proliferation of RATs can be attributed to the fact that existing RATs serve diverse constituencies and deliver substantially differentiated services using a multitude of transport protocols. For instance, NetBus, Socket de Troie and SubSeven use TCP while Back Orifice, DeepThroat and DeltaSource are UDP-based. As RATs evolve they also use different protocols. In this regard, $\mathrm{BO} 2 \mathrm{~K}$ uses both TCP and UDP even though its ancestor BO was exclusively $U D P$-based. The syntax and semantics of client-server messages also demonstrate diverse characteristics. RATs including Back Orifice, SubSeven and BO2K maintain well-formed binary message structures; on the other hand, Trojans such as Dolly and Frenzy follow text-based message formats. Last, some RATs including Eclypse, WanRemote and Drat uses syntax and semantics similar, if not identical, to the standard FTP, HTTP, and Telnet protocols.

In Table II, we show portion of the traffic generated by Eclypse, an FTP-based Trojan and Table V presents traffic generated by WanRemote [43] that clearly follows the HTTP specification in both directions [25]. For brevity, we do not show the HTTP headers of all messages from the server but only the first one in row 2. Server responses are embedded in the data sections of HTTP messages. Table V indicates that the client supports

\begin{tabular}{|c|c|c|c|}
\hline \# & dir & payload & description \\
\hline \multicolumn{4}{|c|}{ protocol: TCP; attacker (denoted as A): 192.168.5.143; victim (denoted as V): 192.168.5.141 } \\
\hline 1 & $\mathrm{~A}: 1071 \longrightarrow \mathrm{V}: 80$ & GET/HTTP/1.1 & standard HTTP msg, implying login \\
\hline 2 & $\mathrm{~V}: 80 \longrightarrow \mathrm{A}: 1071$ & $\begin{array}{l}\text { HTTP/1.0 } 200 \text { OK Content-Type:text/html } \\
<\text { title }>\text { WANRemote } 3.0 \text { - Main Menu }</ \text { title }>\end{array}$ & response with "main menu" to attacker \\
\hline 3 & $\mathrm{~A}: 1089 \longrightarrow \mathrm{V}: 80$ & GET /fm?cd=C:/ HTTP/1.1 & change directory \\
\hline 4 & $\mathrm{~V}: 80 \longrightarrow \mathrm{A}: 1089$ & $<$ title $>$ WANRemote 3.0 - File Manager $</$ title $>$ & enter "file manager" \\
\hline 5 & $\mathrm{~A}: 1105 \longrightarrow \mathrm{V}: 80$ & GET /fm?get=C:/autoexec.bat HTTP/1.1 & get file "autoexec.bat" \\
\hline 6 & $\mathrm{~V}: 80 \longrightarrow \mathrm{A}: 1105$ & WANRemote 3.0 - File Manager: c:/autoexec.bat & file transferred successfully \\
\hline 7 & $\mathrm{~A}: 1142 \longrightarrow \mathrm{V}: 80$ & GET/process HTTP/1.1 & get process list \\
\hline 8 & $\mathrm{~V}: 80 \longrightarrow \mathrm{A}: 1105$ & $<$ title $>$ WANRemote 3.0 - Task List $<$ /title $>$ & return process list \\
\hline 9 & $\mathrm{~A}: 1164 \longrightarrow \mathrm{V}: 80$ & GET /process?kill=800 HTTP/1.1 & kill specified process \\
\hline 10 & $\mathrm{~V}: 80 \longrightarrow \mathrm{A}: 1164$ & $<$ title $>$ WANRemote 3.0 - Task List $</$ title $>$ & processed killed \\
\hline 11 & $\mathrm{~A}: 1175 \longrightarrow \mathrm{V}: 80$ & GET /x-logout HTTP/1.1 & logout from server \\
\hline 12 & $\mathrm{~V}: 80 \longrightarrow \mathrm{A}: 1175$ & $<$ title $>$ WANRemote 3.0 - Log Out $</$ title $>$ & session end \\
\hline
\end{tabular}

Table V. WanRemote(v.3.0)-generated traffic

$\operatorname{HTTP}(v .1 .1)$ and the server uses $\operatorname{HTTP}(v .1 .0)$ forcing each command to be transported using a separate TCP connection. For example, the packet of row 1 has TCP-source port 1071 while the corresponding source ports for messages in rows 3 and 5 are 1089 and 1105 , respectively. By placing different commands in HTTP requests, the client can perform various operations on the victim's machine via the Trojan server. To this effect, the attacker can traverse directories in victim's file system with request " $c d=C: \Gamma^{\prime}$ (row 3 ) and obtain designated files with command " $g e t=C: \rho$ " (row 5). Through "kill=" of row 9, the attacker calls for the termination of a process at the server that reciprocates with status information in row 10. The apparent pattern "WANRemote 3.0" definitely helps detect individual WanRemote(v.3.0) sessions; moreover, the correlation between different sessions (i.e., inter-session correlation) can further improve the detection accuracy.

Drat is a representative of the Telnet-based Trojans in which the server echoes back any attacker input and respective pieces of output as Table VI shows. Acting effectively as a command interpreter, the Drat-server displays the prompt D:/temp $>$ waiting for instructions from the attacker as row 3 indicates. Rows 5, 7 and 9 depict the echoes of the three characters in command dir entered by the attacker in rows 4, 6 and 8; the character-by-character transmission and echo-back mechanism are typical of the Telnet protocol and together provide a reliable way to identify such traffic. We combine together all packets that convey the client's command "run c:/windows/notepad.exe" and show them in the row marked 13-41; the 42-70 line depicts the respective echo activity. Clearly, the attacker mistakenly types ran for the desired run command and later uses "backspace" (0x08) to correct the mistake. By making available editing functionalities, Drat provides a true interactive environment. However, it poses a challenge for security devices that detect 
RATs based on patterns as the latter can be readily evaded by inserting an arbitrary number of edition keys such as backspace, delete, and/or empty spaces. An anti-Trojan system

\begin{tabular}{|c|c|c|c|}
\hline \# & dir & payload & description \\
\hline \multicolumn{4}{|c|}{ protocol: TCP; attacker (denoted as A): 192.168 .5 .143 ; victim (denoted as V): 192.168.5.141 } \\
\hline 1 & $\mathrm{~A}: 1085 \longrightarrow \mathrm{V}: 48$ & |OD 0A & attacker just enter "RETURN". \\
\hline 2 & $\mathrm{~V}: 48 \longrightarrow \mathrm{A}: 1085$ & Welcome to DaRat'z Telnet Rat. & server responses with banner \\
\hline 3 & $\mathrm{~V}: 48 \longrightarrow \mathrm{A}: 1085$ & DRat Version $1.3 .0 \ldots$ DRat : D:/temp > & server displays prompt \\
\hline 4 & $\mathrm{~A}: 1085 \longrightarrow \mathrm{V}: 48$ & $\mathrm{~d}$ & attacker enters cmd (one char at a time) \\
\hline 5 & $\mathrm{~V}: 48 \longrightarrow \mathrm{A}: 1085$ & $\mathrm{~d}$ & server echoes back input \\
\hline 6 & $\mathrm{~A}: 1085 \longrightarrow \mathrm{V}: 48$ & $\mathrm{i}$ & attacker enters next char \\
\hline 7 & $\mathrm{~V}: 48 \longrightarrow \mathrm{A}: 1085$ & $\mathrm{i}$ & server echoes back \\
\hline 8 & $\mathrm{~A}: 1085 \longrightarrow \mathrm{V}: 48$ & $\mathrm{r}$ & attacker enters next char \\
\hline 9 & $\mathrm{~V}: 48 \longrightarrow \mathrm{A}: 1085$ & $\mathrm{r}$ & server echoes back \\
\hline 10 & $\mathrm{~A}: 1085 \longrightarrow \mathrm{V}: 48$ & OD 0A & with "RETURN", cmd "dir" is formed \\
\hline 11 & $\mathrm{~V}: 48 \longrightarrow \mathrm{A}: 1085$ & OD 0A & server echoes back \\
\hline 12 & $\mathrm{~V}: 48 \longrightarrow \mathrm{A}: 1085$ & DRat The Worlds Ultimate Virtual SPY. doc $=46592$ DRAT.exe $\ldots$ & cmd executed, return file list \\
\hline $13-41$ & $\mathrm{~A}: 1085 \longrightarrow \mathrm{V}: 48$ & $\begin{array}{l}\mathrm{ra}|08| \text { un c:/windows/notepad.exe }|0 \mathrm{D} 0 \mathrm{~A}| \\
\end{array}$ & attacker runs an app; " 08 " is backspace \\
\hline $42-70$ & $\mathrm{~V}: 48 \longrightarrow \mathrm{A}: 1085$ & $\begin{array}{l}\mathrm{ra}|08| \text { un c:/windows/notepad.exe }|0 \mathrm{D} 0 \mathrm{~A}| \\
\end{array}$ & server echoes back all inputs \\
\hline 71 & $\mathrm{~V}: 48 \longrightarrow \mathrm{A}: 1085$ & ShellExecute ( 2 Being a Error ) Returned & app is executed; server replies \\
\hline
\end{tabular}

Table VI. Drat(v.1.0)-generated traffic

has to simulate shell functionality and act as a command interpreter, should it successfully identify Telnet-based RATs.

\subsection{Presence of Multiple Evasion Techniques in RATs}

A straightforward approach for RATs to evade detection is to continually change both message structures and banner information as such artifacts are exploited as signatures by firewalls, AVs, and IDSs/IPSs. In this regard, DeepThroat(v1.0) flushes the name of the victim machine (e.g., SHEEP) at the very end of its banner "-Ahhhhhhhhhh My Mouth Is Open SHEEP" while in other versions, the name of the victim machine appears first as demonstrated by the banner 'SHEEP - Ahhhhh My Mouth Is Open (v2)" of DeepThroat(v2.0). Telltale patterns used in DeepThroat change slightly in different versions as well. For instance the pattern Ahhhhhhhhhh in v.1.0 has been shortened to Ahhhhh in versions v.2.0 and v.3.0 and ultimately became $A h h h$ in v.3.1. Such banner adaptations make it more difficult for security systems to detect with a single fixed signature the traffic generated by various versions of DeepThroat operating simultaneously.

Sending decoy messages is another popular evasion technique. By doing so, Trojans such as Doly attempt to induce security systems to generate large numbers of false positives [43], forcing the security officers to spend considerable periods of time examining logs. The latter produces a good chance for the intruders to go undetected. A Doly client attempts to establish a TCP connection with a victim by trying ports $3456,4567,5678,6789$ ", $7890,9182,8374,2345,7654$, and 27559 in sequence four times regardless if a $R A T$ is present. This activity resembles to port scanning which is typically graded as a low-severity surveillance activity by AVs/IDSs/IPSs and is frequently ignored by security officers. Although RATs often operate on their default ports, they can be configured to use ports in either the privileged or non-privileged range. While experimenting with real traffic, we observed that RATs mostly employ port hopping techniques and servers use arbitrary ports selected on the fly readily defeating port-based detection approaches. Hence, a contentbased approach would be by far more fruitful in detecting Trojans using decoy messages, dynamic port hopping, and other evasion techniques. 


\section{A Framework for Apprehending RATs}

Conventional security systems use add-on modules and/or specially-crafted signatures to identify malicious RAT activities and are unaware of RATs unique characteristics. To address these shortcomings, we propose an extensible framework named RAT Catcher that employs network-based detection methods, operates in-line, and manipulates RATtraffic in real-time. We base our design on the following constraints: i) RAT-servers are implanted on victim machines through the channels discussed in Section 3.2. Through its real-time operation, our framework puts emphasis on detecting RAT communications as opposed to conventional AVs and host-based security systems that mainly focus on the Trojan installation process [14]; and ii) features of sought RATs including format of messages, handshake procedures, and functionalities are available typically via reverse engineering, behavior analysis, and data mining techniques [53, 2]. By tracking the progress of all established connections initiated by either normal applications or Trojans, our framework conducts data correlation between different sessions or traffic streams, performs stateful inspection, and identifies abnormal and/or deviating behavior in realtime. Our RAT Catcher stores packets in every data stream, re-assembles them together and the resulting aggregations are subjected to protocol dissection according to standard $T C P / I P$ specifications as well as the syntax and semantics of individual Trojans. In this way, our framework performs layer-7 or application-level inspection and can effectively combat evasive mechanisms used by RATs. As soon as a session is verified as Trojan, our framework can immediately take corrective steps by logging, blocking, terminating the connection or simply taking over the session.

\subsection{Design Rationale and Architecture for the RAT Catcher}

To remotely control a computing system, an attacker should first set up either a TCP or $U D P$ channel with the RAT server implanted on the victim machine. A TCP session is defined by its distinct connection and disconnection processes [15]. The former is a threeway handshake procedure where the initiator (or client) starts a connection with a TCP $S Y N$ packet and the recipient (or server) replies with a TCP SYN-ACK packet which in turn incurs an $A C K$ packet from the initiator. The disconnection procedure typically involves a four-message exchange with each side dispatching a TCP FIN packet and corresponding acknowledgments from the two ends. Evidently, a specific TCP connection can be uniquely identified by the tuple $<$ client-IP, client-port, server-IP, server-port $>$. Similarly, a specific $U D P$ session is also identified by a similar four-element tuple with its termination often designated with a time-out mechanism. Any change in the above tuple implies a new $T C P / U D P$ session. Overall, a connection can be represented with the extended tuple $<$ client-IP, client-port, server-IP, server-port, protocol $>$ where protocol can be either TCP or UDP. Within each session, two data streams exist, one from originator (or client) to recipient (or server) and the second in the opposite direction. Each data stream within a session can be identified with a four element tuple $<I P_{s}, P O R T_{s}, I P_{d}, P O R T_{d}>$, where $I P_{s}$ and $P O R T_{s}$ are the IP address and network port of the source and $I P_{d}$ and $P O R T_{d}$ are their destination counterparts.

Messages exchanged between a Trojan server and client are generated in accordance to the RAT's own syntax rules and semantics and are shipped following the constraints of $T C P / U D P$ transport protocols. The latter may not respect the Trojan's message boundaries, therefore, inconsistencies between Trojan message borders and transport packet demarcations are unavoidable. The transport layer may also deliver packets in an arbitrary order and the original data stream can be only recovered via reassembly by its 
recipient. For instance, the TCP packet of row 5 in Table IV consists of three NetBus Pro messages. Similarly, in the SubSeven-generated traffic of Table VII, multiple Trojan commands are contained in a single TCP packet as row 12 shows. In this, commands IN7 and $C L 7$ open the Webcam of the victim, IN2 and CL2 attempt to open the screen preview and PSS obtains cached passwords. On the other hand, command $\operatorname{dir}\langle C F\rangle\langle L F>$ entered

\begin{tabular}{|c|c|c|c|c|}
\hline \# & timestamp & dir & payload & description \\
\hline \multicolumn{5}{|c|}{ protocol: TCP; attacker (denoted as A): 192.168 .5 .143 ; victim (denoted as V): 192.168 .5 .141} \\
\hline 1 & 0.002077 & $\mathrm{~V}: 55555 \longrightarrow \mathrm{A}: 3689$ & connected. $21: 17 \ldots$, ver: Legends 2.1 & banner from server, access time and version \\
\hline 2 & 20.819971 & $\mathrm{~A}: 3689 \longrightarrow \mathrm{V}: 55555$ & URLhttp://www.fortinet.com/ & instruct victim to goto given "URL" \\
\hline 3 & 20.820263 & $\mathrm{~A}: 3388 \longrightarrow \mathrm{F}: 80$ & (SYN) & connect to server F (i.e., www.fortinet.com) \\
\hline 4 & 21.675816 & $\mathrm{~V}: 55555 \longrightarrow \mathrm{A}: 3689$ & web browser has been opened. & server successfully launches browser \\
\hline 5 & 72.771562 & $\mathrm{~A}: 3689 \longrightarrow \mathrm{V}: 55555$ & FFNF05*.exeC:/ & try to find files "*.exe" under " $\mathrm{C}: / "$ \\
\hline 6 & 72.912758 & $\mathrm{~V}: 55555 \longrightarrow \mathrm{A}: 3689$ & C:/EXPLORER.EXE C:/putty.exe ... & a list of specified files is returned \\
\hline 7 & 108.402655 & $\mathrm{~A}: 3689 \longrightarrow \mathrm{V}: 55555$ & FTPenable!@@@21::1\$\$\$C:/ & enable FTP server by cmd "FTP" \\
\hline 8 & 118.066511 & $\mathrm{~A}: 3689 \longrightarrow \mathrm{V}: 55555$ & FTPdisable & try to disable FTP server \\
\hline 9 & 118.068258 & $\mathrm{~V}: 55555 \longrightarrow \mathrm{A}: 3689$ & FTP server disabled & remote FTP server is disabled \\
\hline 10 & 129.070129 & $\mathrm{~A}: 3689 \longrightarrow \mathrm{V}: 55555$ & IRG & access registry editor \\
\hline 11 & 129.131769 & $\mathrm{~V}: 55555 \longrightarrow \mathrm{A}: 3689$ & Console Control Panel Environment ... & menu of registry editor is returned \\
\hline 12 & 139.577822 & $\mathrm{~A}: 3689 \longrightarrow \mathrm{V}: 55555$ & IN7CL7IN2CL2PSS & open Webcam (IN7CL7), get passwords (PSS) \\
\hline 13 & 139.579805 & $\mathrm{~V}: 55555 \longrightarrow \mathrm{A}: 3689$ & PSS cached passwords: [www.fort...] & return network addr, login name, password \\
\hline 14 & 208.272307 & $\mathrm{~A}: 3689 \longrightarrow \mathrm{V}: 55555$ & CLG & close registry editor \\
\hline
\end{tabular}

Table VII. SubSeven (v.2.1)-generated traffic

by the Drat attacker in Table VI is split into four TCP packets; the payload of each packet contains only one or two characters.

There are often multiple concurrent sessions between a RAT client and its server coexisting with sessions created by other applications. For instance in the SubSeven traffic of Table VII, two sessions coexist after the packet in row 3. The first is identified by tuple $\langle A, 3689, V, 55555, T C P>$ where $A$ stands for the attacker located at $I P$ address 192.168.5.143 and $V$ is the victim with $I P$ address 192.168.5.141. The second session is identified as $\langle A, 3388, F, 80, T C P>$ where $F$ is the $I P$ address of the Web server WWw.fortinet.com. In a similar fashion, the DeepThroat traffic of Table VIII creates two sessions, the control session identified as $\langle A, 60000, V, 2140, U D P>$ and the data session commencing at row 4 and identified as $\langle A, 60000, V, 3150, U D P\rangle$. Most anti-Trojan systems detect $R A T$ control-sessions only and they are completely blind to respective data-sessions simply because the latter lack unique telltale patterns.

\begin{tabular}{|c|c|c|c|}
\hline \# & dir & payload & description \\
\hline \multicolumn{4}{|c|}{ protocol: UDP; attacker (denoted as A): 192.168 .5 .143 ; victim (denoted as V): 192.168 .5 .141} \\
\hline 1 & $\mathrm{~A}: 60000 \longrightarrow \mathrm{V}: 2140$ & 00 & a "ping" message \\
\hline 2 & $\mathrm{~V}: 2140 \longrightarrow \mathrm{A}: 60000$ & host - Ahhhh My Mouth Is Open (v3.1) & a "pong" message \\
\hline 3 & $\mathrm{~A}: 60000 \longrightarrow \mathrm{V}: 2140$ & 39 & a request to "create directory" \\
\hline 4 & $\mathrm{~A}: 60000 \longrightarrow \mathrm{V}: 3150$ & c:/temp/host & parameter to "create directory" command \\
\hline 5 & $\mathrm{~V}: 2140 \longrightarrow \mathrm{A}: 60000$ & Directory Created & reply to command "create directory" (39) \\
\hline 6 & $\mathrm{~A}: 60000 \longrightarrow \mathrm{V}: 2140$ & 35 & command "freeze mouse" \\
\hline 7 & $\mathrm{~V}: 2140 \longrightarrow \mathrm{A}: 60000$ & Mouse frozen & reply to command "freeze mouse" (i.e., 35) \\
\hline 8 & $\mathrm{~A}: 60000 \longrightarrow \mathrm{V}: 2140$ & 12 & command "send host info" \\
\hline 9 & $\mathrm{~A}: 60000 \longrightarrow \mathrm{V}: 3150$ & http://www.fortinet.com & parameter to "send host info" command \\
\hline 10 & $\mathrm{~V}: 2140 \longrightarrow \mathrm{A}: 60000$ & Host Sent To URL & reply to command "send host info" (12) \\
\hline
\end{tabular}

Table VIII. DeepThroat(v.3.1)-generated traffic

For all established connections originated by either normal applications and/or suspected-Trojans, RAT Catcher maintains session records, including information of connection initiator and recipient, messages exchanged, and application type of the session. Such session information is not only maintained during the lifetime of a session but also remains accessible beyond its lifetime as part of a history repository. The capability of tracking the state of each active session facilitates stateful inspection, intra-session data fusion, and inter-session correlation for Trojan-generated traffic. With the help of stored information, we can also determine the progress of a session. For instance, information on connection status can indicate whether a TCP session is in the three-way handshake procedure, has established connection, or is at its termination phase. By correlating the data of streams within the same session, RAT Catcher can rapidly determine whether an 


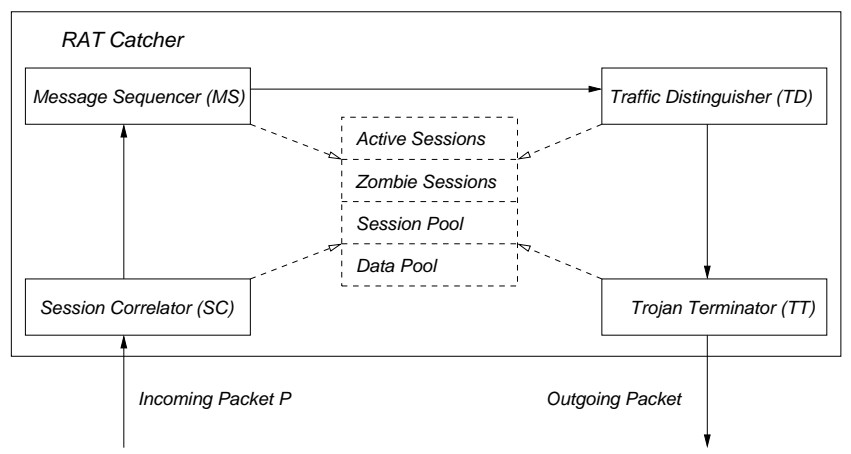

Figure 3. RAT Catcher architecture

attacker's operations are successful. In addition, by correlating the information from prior sessions with an ongoing connection, a RAT data channel could be readily associated with its control channel, thereby prompting the same preventive/punitive action by the RAT Catcher. Furthermore, we use the information of defunct sessions stored in the history repository for carrying out session correlation computations that help determine the traffic type of currently active sessions.

The storage of all transport packets facilitates their remapping to Trojan messages by the packet re-assembly procedure that takes place with the help of the syntax and semantics of known Trojan protocols. The objective of this aggregation also known as Message Sequencing, is to conceal the packet demarcation imposed by the transport layer and restore the boundaries of Trojan messages. Without sequencing, Trojan sessions may go undetected if their constituent messages happen to span multiple transport packets. Given the large number of existing RATs and the variety of protocols used, it becomes challenging to identify a RAT session effectively and efficiently. To classify data streams, the RAT Catcher resorts to multiple techniques that include traffic correlation, application protocol analysis, and stateful inspections in addition to fine-tuned signatures. Once a session is confirmed as Trojan, our framework may immediately block, terminate or take over the session besides log generation. Figure 3 shows the architecture of our framework that entails the following modules: Session Correlator (SC), Message Sequencer (MS), Traffic Distinguisher (TD), and Trojan Terminator (TT).

Once a packet $P$ arrives at the RAT Catcher, the Session Correlator (SC) module determines whether there exists a session $S$ in which $P$ belongs to; if there is none, a new session $S$ is created for $P$. Based on information about $S$ or correlation with other active or defunct sessions, it may be possible to immediately determine whether $P$ is part of a $R A T$ such as Back Orifice, SubSeven, NetBus, or DeepThroat. $P$ is then handed over to the Message Sequencer $(M S)$ along with its session information $S$, where $P$ is re-assembled with other existing packets of the same stream to form a sequence of application messages. In turn, this message sequence is transferred to the Traffic Distinguisher (TD) to determine the specific application type of the session. Finally, $P$ arrives at the Trojan Terminator (TT) where information on $S$ is appropriately updated, $P$ is stored in $S$ to help forthcoming re-assembly efforts and a corrective action may be taken if RAT traffic has been found.

\subsection{Session Correlator (SC)}

To maintain information for each session, we use the session data structure whose key fields are shown in first part of Table IX. Each connection is assigned a session structure and is uniquely identified by its first five fields: source and destination $I P$ addresses (SIP, DIP), 
source and destination port numbers (SPORT, DPORT) and protocol (PROTO). Field TYPE indicates the application type of the session such as DeepThroat, NetBus, WanRemote etc.; this field can assume the value bypass, should the application type cannot be determined after a certain amount of traffic in the session has been inspected or the session is generated by a normal application. The field CONFIRM indicates whether the value in TYPE has been derived from correlating streams in both directions of the same session, obtained by association with other sessions (active or zombie), or simply drawn based on different messages in a uni-directional traffic. Clearly, should a session application type be drawn using multiple criteria -data streams, sessions, and correlations- the classification accuracy is improved. The two data streams in a session, the one from client to server and its reverse counterpart are stored in fields CLIENT and SERVER respectively; these pointers to stream structures are discussed in Section 4.3.

\begin{tabular}{|c|c|c|}
\hline field name & size (bytes) & description \\
\hline \multicolumn{3}{|r|}{ Key fields of the session data structure } \\
\hline SIP & $\frac{4}{4}$ & $I P$ address of the host at one end of the connection \\
\hline DIP & 4 & $I P$ address of the host at the other end of the connection \\
\hline SPORT & 4 & port number of the host with IP address SIP; may assume special value of unknown \\
\hline DPORT & 4 & port number of the host with $I P$ address DIP; may assume special value of unknown \\
\hline PROTO & 1 & protocol utilized by the session (TCP or $U D P$ ) \\
\hline TYPE & 4 & identify traffic type, such as DeepThroat, NetBus, SubSeven; can be unknown \\
\hline CONFIRM & 4 & TYPE is drawn from uni-or bi-directional streams, intra- or inter-session correlations \\
\hline SERVER & 4 & pointer to server stream data structure \\
\hline CLIENT & 4 & pointer to client stream data structure \\
\hline \multicolumn{3}{|r|}{ Key fields of the stream data structure } \\
\hline $\begin{array}{ll}\text { state } \\
\text { next-seg }\end{array}$ & $\begin{array}{l}4 \\
4\end{array}$ & state of the stream (TCP only) such as CLOSED, LISTEN, SYN_SENT, SYN_RCVD, ESTABLISHED \\
\hline ISN & $\begin{array}{l}4 \\
4\end{array}$ & $\begin{array}{l}\text { next sequence number expected ( } T C P \text { only), computed based on information of sender } \\
\text { initial sequence number of the current stream ( } T C P \text { only) }\end{array}$ \\
\hline & 4 & pointer to the root of interval-tree storing all packets of the stream \\
\hline total-size & 4 & total size of data transferred in the stream so far (UDP only) \\
\hline data-size & 4 & number of bytes stored in "data" buffer (UDP only) \\
\hline
\end{tabular}

Table IX. Key fields in data structures session and stream

Figure 4 shows an hierarchical structure termed active sessions table that we use to organize session pertinent information; it provides efficient session insertions, retrievals, deletions, and facilitates intra-session and inter-session correlations. We first use a hashtable to group sessions with hash function $H(S I P, D I P, P R O T O)=((h>>16)$ xor $(h>>8))$ $\bmod h_{\text {size }}$ where auxiliary function $h$ is defined as $h=($ SIP xorDIP xorPROTO), " $>>$ " is the right shift operation, and h_size is the size of the hash table. In computing the hash value, SIP, DIP, and PROTO are treated as integers with protocols TCP and UDP assuming values $0 x 06$ and $0 x 11$ respectively. Although simple, function $H$ has exhibited near-uniform distribution in our experiments. Next, each hash-table entry points to a splay tree $T$ that complies with the binary search tree property and attains an amortized time by moving a tree node closer to the root every time it is accessed [48]. Clearly, frequently accessed elements are more likely to be closer to the root.

A splay tree $T$ anchored off each entry of the hash table helps organize all sessions that present the same hash value. In our framework, each node in $T$ represents all connections established between a source/destination pair described by fields SIP, DIP and PROTO of the structure session (of Table IX). More specifically, every node of $T$ is associated with a port mapper consisting of two tables; one organizes the ports (SPORT) used by SIP and the other stores ports (DPORT) used by DIP. If a port number is active and occupied by a session, the appropriate record for the session is stored in the corresponding slot of the port mapper. Multiple connections sharing the same port number are organized with a linked-list under the slot indicated by the port number. Finally, all session specific data are maintained in the session pool of Figure 4 and are organized as linked-lists as well.

To facilitate session retrieval, we designed function session-find $(P$, wildcard $)$ whose goal is to locate the session $S$ that packet $P$ belongs to. This function initially searches the active session table with the help of data in tuple $<$ SIP, SPORT, DIP, DPORT, PROTO $>$ available through $P$. If the outcome is an existing session $S, P$ is client-initiated and $S$ is marked by $S C$ as having forward direction "DIR: forward"; otherwise, $S C$ looks up the 


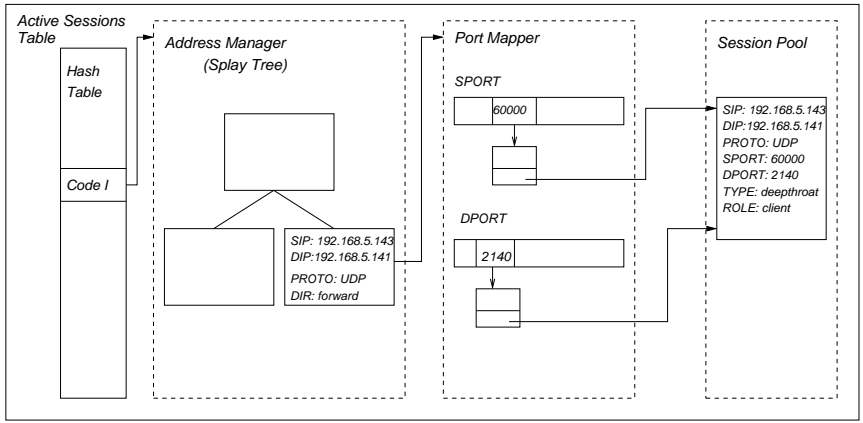

Figure 4. Session information before data channel is established for traffic in Table VIII

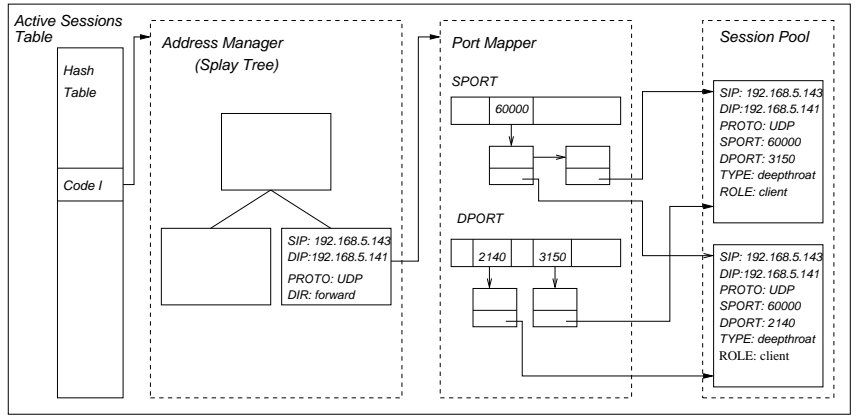

Figure 5. Session information after data channel is established for traffic in Table VIII

active session table again with a new tuple $<D I P, D P O R T$, SIP, SPORT, PROTO $>$ formed by exchanging the roles of source and destination ends. A non-empty result $S$ in this second attempt indicates that $P$ is part of a server-originated stream tagged with direction " $D I R$ : reverse". The wildcard argument of session-find() specifies whether wildcard matching is conducted in the session lookup. By assuming value "source-port" or "destination-port" for parameter wildcard, session-find() considers any active source or destination port to be a match. Should wildcard be designated as "none", an exact port match is performed.

Algorithm 1 outlines the three-step operation $S C$ carries out. First, the function call session-find( $P$,none) is used to extract the session $S$ corresponding to packet $P$ by traversing the active session table; a non-null $S$ indicates that $P$ belongs to an existing session and $S C$ simply exits by returning $S$. Next, $S C$ verifies whether $P$ initiates a new session that acts as a data channel associated with an existent RAT control channel. Finally, the application type of the newly created session $S$ for packet $P$ is set by correlating $S$ with active/defunct session tables.

We use the DeepThroat traffic of Table VIII as an example to describe the procedure followed by $S C$. The traffic segment indicates that the attacker establishes two UDP sessions with 2140 and 3150 as their corresponding destination ports; the former acts as the command channel and the latter as the data channel. By routing the traffic of Table VIII to our RAT Catcher, SC creates session $<A, 60000, V, 2140, U D P>$ immediately after packet at row 1 is encountered. When $S C$ deals with packet $P$ of row 2 , the invocation of session-find( $P$, none) yields session $\langle A, 60000, V, 2140, U D P\rangle$ whose mark "DIR: reverse" indicates $P$ to be a server-originating packet. We assume that after processing the first two packets, the $S C$ records information on session $\langle A, 60000, V, 2140, U D P\rangle$, 


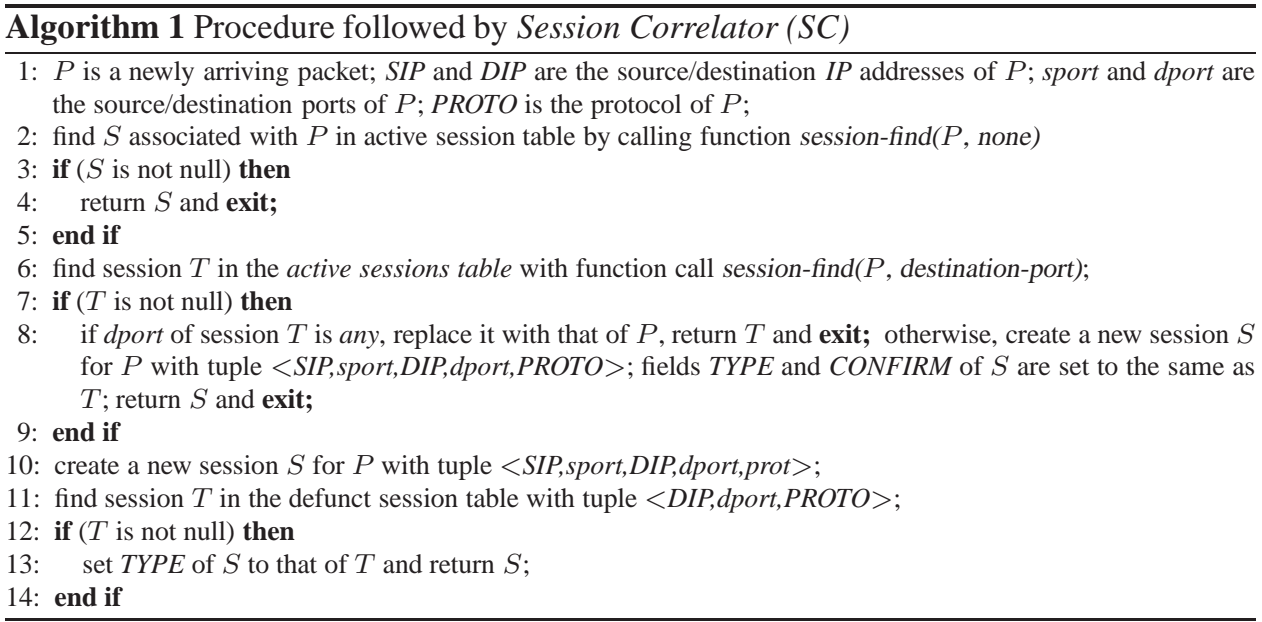

constructs the session table of Figure 4 and tentatively marks the session as DeepThroat. When the packet in row 4 is encountered, the RAT Catcher establishes that no session $<A, 60000, V, 3150, U D P>$ exists as evidenced by Figure 4. Before creating a new session $<A, 60000, V, 3150, U D P>, S C$ looks up in the active sessions table for any session matching $<A, 60000$, V,any, $U D P>$ with the help of the call session-find( $P$, destination-port). By checking the type of the session in the lookup result, $\angle A, 60000, V, 2140, U D P>$, the $R A T$ Catcher establishes that a DeepThroat control channel indeed exists and therefore marks the newly created session as DeepThroat (Figure 5). Algorithm 1 carries out this session correlation between RAT control and data channels in lines 6 to 9 .

Information about terminated or zombie sessions, especially those recently torn down, may be helpful to determine the application type of currently active sessions. For example, if a service provided on a specific network port of a host identified with the tuple $<$ DIP,DPORT,PROTO $>$ has been determined as a Trojan server by some previous sessions, its application type is not expected to change abruptly. Based on the above observation, our RAT Catcher keeps information about zombie sessions of Trojan servers accessible for a period of time -configurable but set to 5 minutes by default- and such zombie sessions are organized in the defunct session table with key of $\langle D I P, D P O R T, P R O T O\rangle$ and value of TYPE. Any time a new session $\angle S I P, S P O R T, D I P, D P O R T, P R O T O>$, is generated by the $S C$, the defunct session table is consulted with query $\langle D I P, D P O R T, P R O T O\rangle$, and the application type of the returned session, if any, is assigned to the newly created session. For instance, by the time RAT Catcher processes the connection beginning at row 3 of Table V, session $\langle A, 1071, V, 80, T C P\rangle$ has become zombie and therefore, it is stored in the defunct session table. By simply searching for $\langle V, 80, T C P\rangle$ in the defunct session table, the application type of the session established by row 3 of Table $\mathrm{V}$ can be quickly determined as WanRemote. Such a correlation between ongoing connections and alreadyknown zombies can establish relevant temporal session associations and rapidly identify the application types of ongoing sessions.

\section{3. $\quad$ Message Sequencer (MS)}

We design the stream structure -key elements of which appear in the lower part of Table IX- so that packet re-assembly and state tracking for a data stream can be easily carried out. Different types of information are stored for connection-oriented and connectionless channels. For TCP streams, field state tracks the connection state of its originator and can be SYN-SENT, SYN-RCVD, ESTABLISHED, or CLOSE; fields ISN and next-seq maintain the initial sequence number and the next expected sequence number, respectively. Last, data is a pointer to an interval-tree [17] used to organize all encountered 
stream packets according to a search key $\left[n_{1}, n_{2}\right]$ where $n_{1}, n_{2}$ represent start and end sequence numbers; the value of every interval-tree node is a single packet of the stream in question. In a $U D P$ stream, the data points to a buffer that stores data received but unprocessed for the stream in question thus far, whose size is indicated by field data-size. The field total-size indicates the number of bytes transmitted helping track the volume of data encountered by the session. We define function call stream-find $(S, P)$ to retrieve the stream $I$ corresponding to packet $P$ within session $S$.

The Message Sequencer uses an interval-tree $I$, implemented as a red-black tree to store stream-pertinent data. The key $\left[V_{s}, V_{e}\right]$ of a specific node $V$ in $I$ represents the start and end sequence numbers ( $S S N$ and $E S N$ ) of the corresponding packet $P ; V_{s}$ can be directly obtained from field sequence number of $P$ 's TCP header while $V_{e}$ can be derived with the help of the fields total length, IP header length as well as TCP header size of the packet. For $U D P$ streams, we simply assign the current value in total-size of the stream as the start sequence number $(S S N)$ of the incoming packet and its $E S N$ can be derived from $S S N$ and the packet size. For any two packets $P$ and $Q$ of the same stream, we define different relationships based on their sequence intervals. By denoting $P_{s}$ and $P_{e}$ as the start and end sequence numbers for $P$ as well as $Q_{s}$ and $Q_{e}$ for $Q$ respectively, we can determine that: a) $Q$ is a duplicate of $P$ if $P_{s}=Q_{s}$ and $P_{e}=Q_{e}$; b) $P$ and $Q$ overlap if $P_{s}<Q_{s}$ and $Q_{s}<P_{e}<Q_{e}$ or $Q_{s}<P_{s}$ and $P_{s}<Q_{e}<P_{e}$; c) $P$ contains $Q$ if $Q_{s}>P_{s}$ and $Q_{e}<P_{e}$ or $Q$ contains $P$ if $P_{s}>Q_{s}$ and $P_{e}<Q_{e}$; and d) $P$ precedes or follows $Q$ if $P_{e}<Q_{s}$ or $P_{s}>Q_{e}$, respectively. With the help of these interval relations, we design functions to manipulate the interval tree: interval-insert $(I, P)$ inserts a node representing packet $P$ into $I$, interval-delete $(I, P)$ removes the node of packet $P$ from $I$, and interval-retrieve $(I, P)$ returns a pointer to a node $Q$ of the tree $I$ provided that a duplicate, overlap, or containment relationship between $Q$ and $P$ can be established; otherwise, the outcome is a NULL. Evidently, these operations maintain complexity $O(\log (n))$ where $n$ is the number of nodes in $I$. In addition, the function packet-build(I, SSN, ESN) creates and returns a new packet $Q$ with interval indicated by [SSN, ESN]. Finally, function interval-traversal( $I$ ) performs an in-order tree walk of $I$ and lists all intervals (i.e., packets) in sorted order by their $S S N$; this function is useful for logging packets into permanent storage.

The stream re-assembly process used by $M S$ is presented in Algorithm 2 and works as follows: for an arriving packet $P, M S$ obtains the session $S$ of $P$ and function streamfind $(S, P)$ is invoked to fetch its stream $I$. Next, $M S$ checks the freshness of $P$ by calling

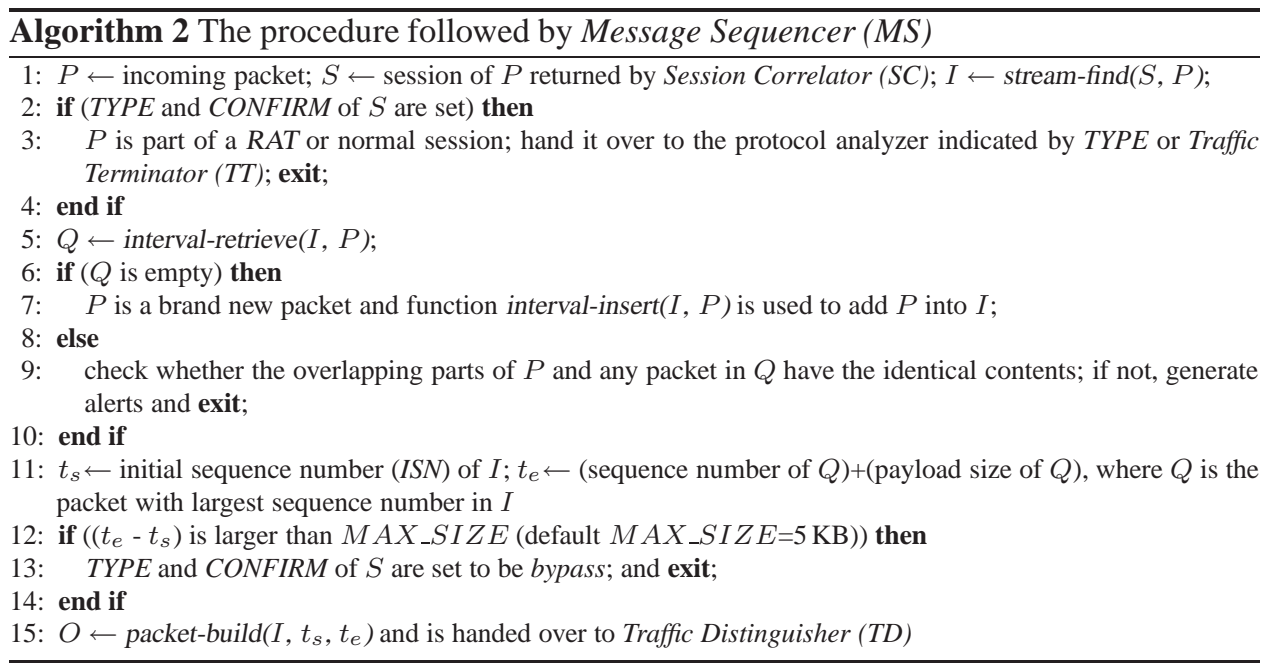

function interval-retrieve $(I, P)$, which returns a pointer to node $Q$ of tree $I$. A NULL $Q$ implies that $P$ is a new packet and can be inserted into $I$ with the help of function interval-insert $(I, P)$. If $P$ and $Q$ are duplicates, their payloads are compared to ensure that 


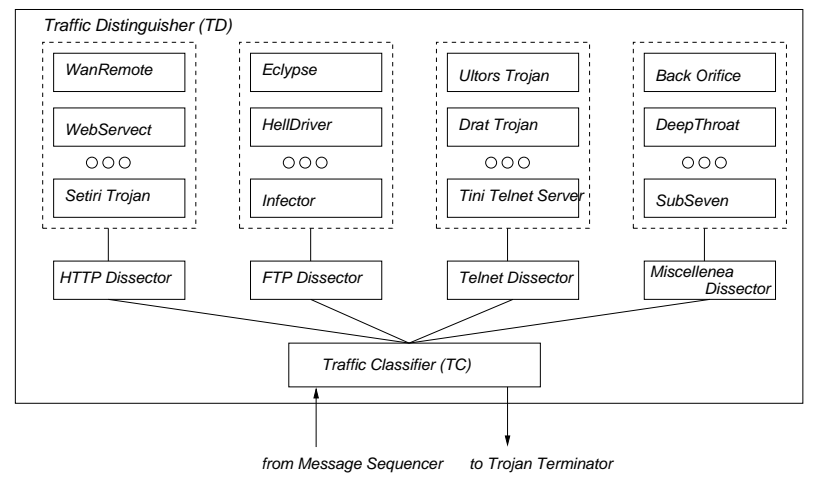

Figure 6. Traffic Distinguisher (TD) components

they have identical content before $P$ is inserted into $I$ with a different timestamp. In a similar manner, for cases where $P$ and $Q$ overlap and either $P$ contains $Q$ or $Q$ contains $P$, their contents on the common sequence interval are compared; if they share the same content for the overlapping part, $P$ is inserted into $I$ since $P$ is a normal overlap packet or retransmission of $Q$; otherwise, $P$ is suspicious as it may have been crafted with evasive tools $\|$ and administrator-specified counter measures such as dropping can be applied. Finally, function packet-build( $I, S S N, E S N)$ is called, with $S S N$ the initial sequence number of the stream $I$ and $E S N$ the largest sequence number thus far in $I$, to re-assemble the data stream and the resulting aggregation, termed super-packet $O$, is handed to the Traffic Distinguisher (TD) module.

\subsection{Traffic Distinguisher (TD)}

As every Trojan follows its own protocol, it would be unrealistic to use a monolithic mechanism for detecting all possible RATs. The Traffic Distinguisher (TD) module addresses this challenge by using a multi-phase traffic classification scheme. First, all incoming traffic is categorized by the Traffic Classifier (TC) into four general types of streams: HTTP, FTP, Telnet and Miscellanea. Subsequently, each type is handled by its own traffic Dissector. In the Miscellanea Dissector, we employ specific Trojan analyzers to detect streams that potentially belong to RATs using proprietary protocols as such Trojans hardly manifest any commonalities. Figure 6 shows the TD components and their organization. Traffic Classifier (TC) component mainly employs heuristic rules to classify traffic. For every incoming packet $P$, along with its session $S$, stream $I$, and the superpacket $O$ constructed by module Message Sequencer $(M S), T C$ determines the traffic type of $P$ with the help of the following rules:

1. packet $P$ is client-initiated: The super-packet $O$ is matched against pattern "method URL HTTP/[0.9|1.0|1.1]", where method is any legitimate HTTP-method such as GET, POST, and PUT [25]; a positive result causes session $S$ to be marked as HTTP. Similarly, $S$ is claimed as FTP-compliant traffic if $O$ follows pattern "command parameter $|O D O A|$ ". $S$ is marked as Telnet-compliant traffic if application payload of $P$ is less than 3 bytes and $O$ follows pattern "command parameter $|O D O A|$ ". Otherwise, $S$ is marked as Miscellanea. 
2. packet $P$ is server-initiated: If $O$ follows pattern "HTTP/[0.9|1.0|1.1] 3-digits statustext", $S$ is marked as HTTP-compliant traffic. $S$ is deemed as FTP traffic if $O$ conforms with syntax "text-string $|O D O A|$ ". In order to identify Telnet-compliant traffic, $O$ is first split into lines with demarcation symbols $|O D O A|$, the first line is then rewritten by simulating the effects of function-keys "backspace" and "delete", and the outcome is compared against the client-dispatched command. If all above checks fail, $S$ is marked as Miscellanea.

Once $T C$ determines the type of $P$, it invokes the corresponding Dissector.

The Dissectors analyze the header and body of exchanged messages in both directions of a session and correlate messages within the same session to further improve classification accuracy. In particular, the HTTP Dissector inspects the header and body of HTTP messages, attempting to identify RATs that follow the HTTP protocol such as WANRemote. The FTP Dissector identifies FTP-based Trojans including Eclypse, HellDriver and Infector while the Telnet Dissector detects the Drat, Ultors and Tini Telnet Server Trojans that follow Telnet protocol specifications. Similarly, RATs including SubSeven and NetBus that use their own protocol specifications are handled by Miscellanea Dissector; the latter is mostly based on analyzers that use RAT specifications often obtained through reverse engineering.

By and large, RAT streams that are transported via the same protocol appear to be very similar except in their use of ports, banners, and server-replies received. For instance, all FTP-based Trojans such as Eclypse (Table II) and HellDriver use TCP-ports for their control and data channels and their server use banners that differ only in content. For example, the Eclypse banner is "220 EclYpse 's FTP Server is happy to see u !" and that of HellDriver's is "220 ICS FTP Server ready". Although FTP-compliant RATssupport different command sets, they overall follow the FTP specification. Based on these observations, our RAT Catcher detects FTP-compliant RAT types by mainly using their server-banners and client-command sets. As soon as the banner and command set of a newly-established FTP-based RAT become available, RAT Catcher can successfully identify the Trojan in question through proper augmentation of the FTP Dissector. This also applied to HTTP and Telnet-compliant RATs as well. Algorithm 3 shows the overall operation of the FTP Dissector; the dissectors for Telnet and HTTP are laid out similarly. Algorithm 3 treats

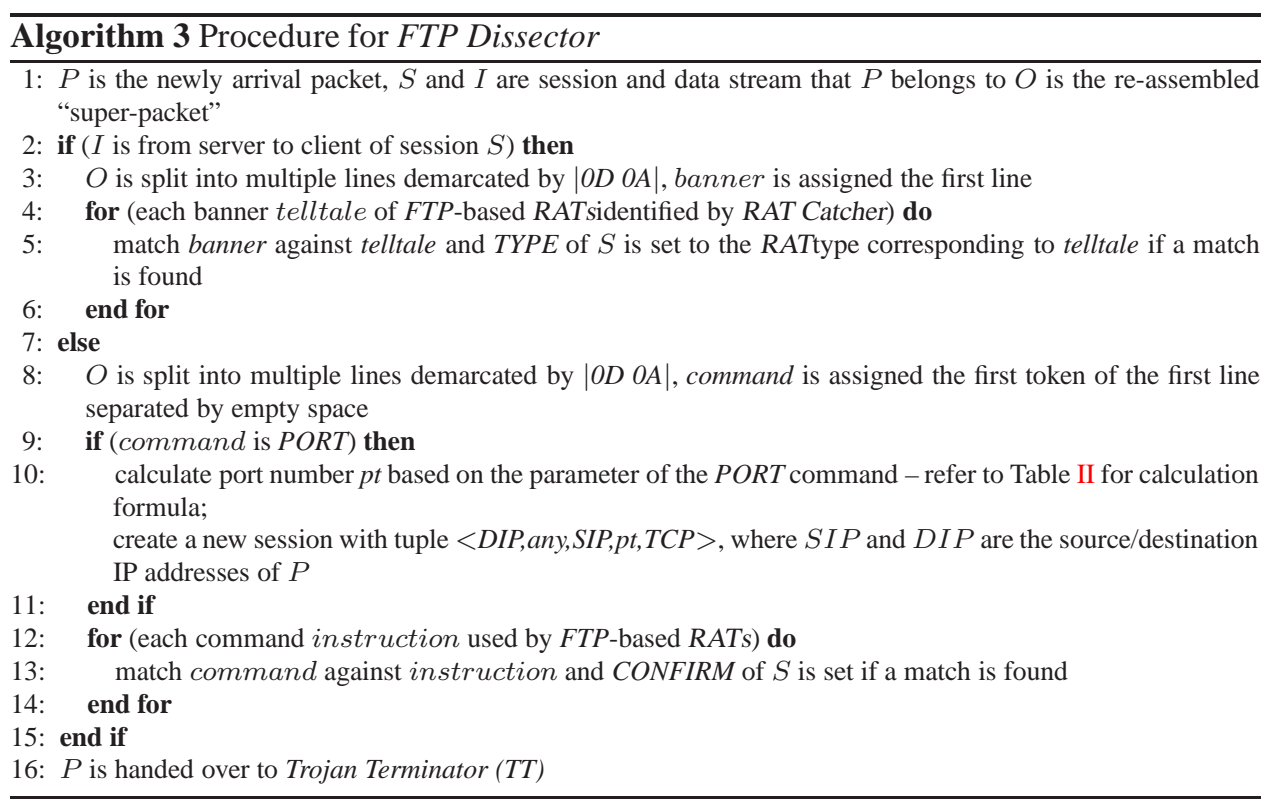

FTP command PORT in a special way as this command specifies the port used for the data channel. A pseudo-session $\langle D I P$, any,SIP,pt,TCP $>$ is created where $S I P, D I P$ are the source/destination IP addresses of the packet in question, and $p t$ is the port number 
specified in command PORT. As the network port that would be used by Trojan server data channel in the near future is yet unknown, a placeholder any is used instead.

The function of Miscellanea Dissector is inherently different from that of its counterparts in Figure 6 as it attempts to detect proprietary RAT communication protocols that hardly demonstrate any commonalities. This dissector essentially acts as a scheduler for all registered analyzers whose objective is to dissect exclusively proprietary protocols. For each incoming packet $P$, Miscellanea Dissector invokes in sequence all RAT-analyzers that have been implemented and incorporated in our framework. The process continues until the application type of $P$ is either determined or all $R A T$-analyzers have been used with no outcome.

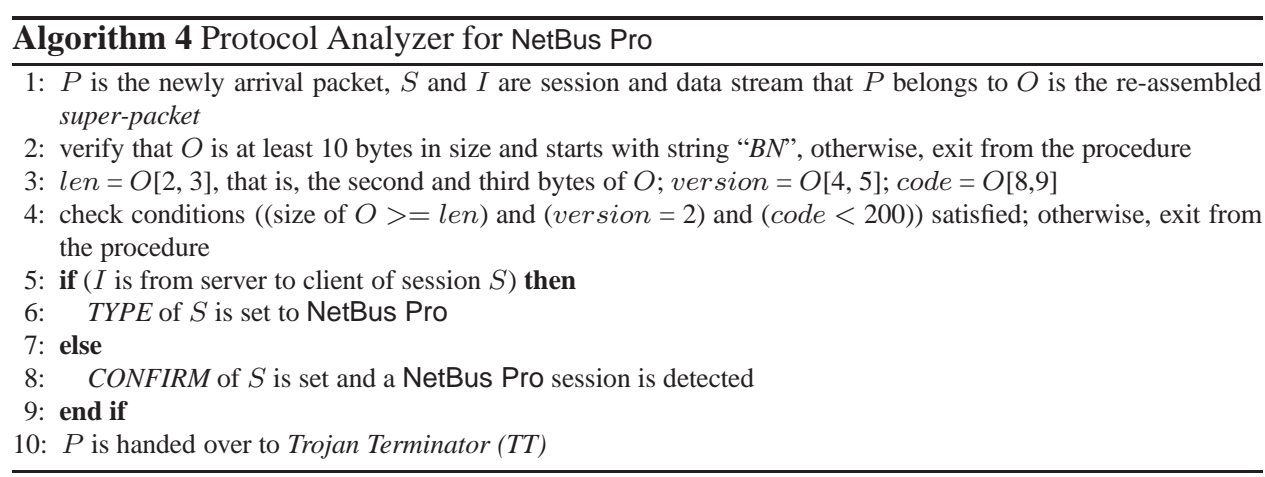

Analyzers within the Miscellanea Dissector share similar working mechanisms and Algorithm 4 shows the skeleton of the NetBus Pro analyzer. In brief, Algorithm 4 first finds boundaries of $R A T$-messages from the re-assembled super-packet $O$ provided by module $M S$. In particular, Algorithm 4 inspects whether super-packet $O$ satisfies the minimum message size of 10 Bytes and starts with telltale " $B N$ " as necessitated by NetBus Pro specification. It then examines whether the restored messages yield NetBus Pro traffic. This is done by extracting well-defined fields including message-size, version-number, and command-code and tentatively marking the flow as NetBus Pro. Finally, Algorithm 4 uses the first server-message to confirm the initial marking by the first client message and set the field CONFIRM.

\subsection{Trojan Terminator (TT)}

The Trojan Terminator (TT) module of the RAT Catcher allows for counter measures to be taken for different types of detected RATs. The TT module examines the application type of a received packet $P$ in conjunction with the status of its session $S$ and stream $I$ and take administrator-specified actions for the various types of traffic. If fields $T Y P E$ and CONFIRM of session $S$ are not set, TT simply forwards $P$ to the next hop enroute to its destination. Otherwise, TT uses a number of options including alert generation, logging of $P$ as well as its data stream $I$ and session $S$, blocking of subsequent messages from the same session, and/or take-over by acting as a RAT server to the initiator of the session. Information shown in Table IX for session $S$ is also updated based on $P$ to help subsequent re-assembly operations and improve the accuracy of RAT Catcher.

In addition, TT can become more proactive by disabling identified RATs. In particular, it may remove all Trojan-related components from the victim's file system. For instance, NetSphere has a unique feature that allows for the purging of its server through the client-command KillServer. The command $<$ KillServer $>$ issued by the NetSphere client forces the server to disconnect itself from the network; in addition, the server un-installs itself from the victim system by removing all NetSphere pertinent files. Similarly, Trojan GateCrasher also provides commands "uninstall;" and "end;" to terminate the execution of servers and purge from victim machines all pertinent files. By simulating the roles of 
RAT-clients, our TT can take over confirmed Trojan sessions and purge RATs from victim machines. In the same manner, our $T T$ can play the role of the RAT-servers, helping collect vital information about attackers without suffering the destructive consequences of RATservers. In order to take over a detected RAT session, the TT sends a TCP RESET packet or an ICMP destination unreachable message to the server. Subsequently, $T T$ crafts fake replies for all client-generated commands, and record all input from the attackers. Finally, it is worth pointing out that differentiated actions can be taken according not only on the $R A T$ types but also on the transport protocols.

\section{Experimental Evaluation of the RAT Catcher}

We have implemented the proposed RAT Catcher in C as a subsystem in the IPS-module of FortiGate, a multi-function security protection system and a standalone network device providing firewall, AV, and IDS/IPS functionalities [32]. The modular architecture of FortiGate forms the basis for its extensibility and scalability, allowing for the seamless coupling of all our RAT Catcher-related components. In our experiments we used FortiGate-300 that operates in inline fashion, has 2 Gigabyte main memory, can manage prorated $400 \mathrm{Mbps}$ traffic, and maintains upto one million concurrent network connections. We subjected the RAT Catcher to a wide range of experiments based on the testbed shown in Figure 7 with a number of test machines undertaking the roles of either $R A T$-servers or clients. Test machines run either Linux or Windows as a large number of RAT-clients and servers are available for these platforms. All test machines were connected to the FortiGate300 via two switches supporting 100/1,000 Mbps ports: the first simulates the internal network where $R A T$-servers are found while the second switch plays the role of external network where various $R A T$-clients are operated by attackers. To verify the behavior of our RAT Catcher, we installed Ethereal traffic sniffers [23] -denoted as Sniffers in Figure 7to capture data exchanges among RAT-clients, RAT Catcher and RAT-servers. In what follows, we report on our laboratory-based effort to establish the accurate operation of the RAT Catcher and baseline performance characteristics. We also report on the deployment and performance of the framework in actual networks.

\subsection{Accuracy on $R A T$ Detection}

Our initial focus was on establishing the accuracy of our RAT Catcher in detecting Trojans that use either the HTTP, FTP, Telnet standard specifications or proprietary protocols with the help of the test environment of Figure 7. We also intended to compare the behavior of RAT Catcher versus that of Snort, an open-source IPS that predominantly uses signatures for RAT detection [44]. In [9], we discuss how Snort can be used for this purpose and outline specific rules. Every time we implanted a new Trojan server or client on a test machine, we re-installed the OS, network and regular applications of the machine in question to avoid any accidental interference. For all experiments we discuss in this section, the action taken by RAT Catcher on identified Trojan sessions is configured to be pass, meaning that the RAT Catcher only generates alerts for the detected RAT sessions and simply forwards all traffic. We proceed with our experiments in three stages with different test procedures: manual test, automated test, and tests designed specially for encrypted RATs.

In the manual testing stage, we initially installed all FTP-based Trojan servers identified by our RAT Catcher, including Eclypse, HellDriver, and Infector, then manually execute their corresponding clients and enter randomly selected RAT commands. Our RAT Catcher successfully identified all such Trojan communications and generated appropriate alerts. Next, we installed a subset of RAT servers of different types on a single test machine denoted as $A$, carefully configured each of them to avoid any conflict on communication ports, installation locations, clashing file modifications, and activated them 


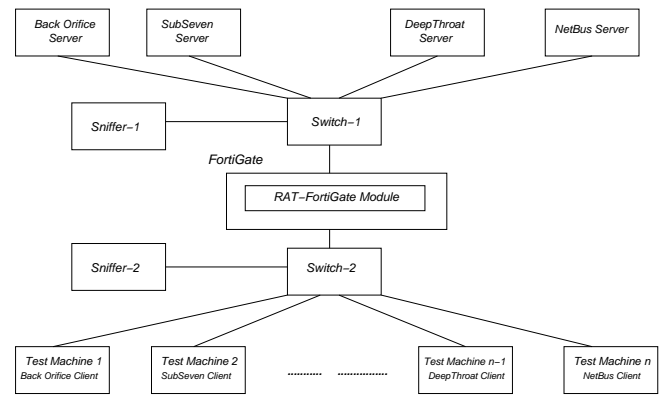

Figure 7. Testbed for RAT Catcher subject to real traffic

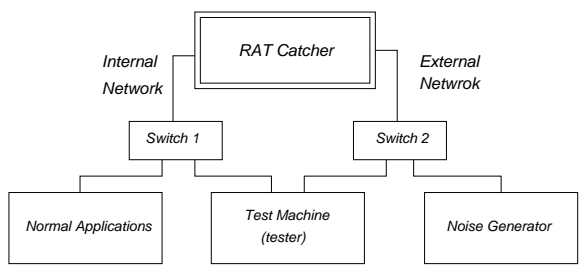

Figure 8. Testbed for RAT Catcher subject to synthetic traffic

all simultaneously. By executing $R A T$ client programs one at a time to communicate with their own servers on test machine $A$, our RAT Catcher revealed all Trojan activity. We observed the same accurate detection from the RAT Catcher when multiple RAT-clients were invoked at the same time. The RAT Catcher still achieved a perfect detection accuracy even when background or attack-free traffic generated by WU-FTPD co-existed during the testing. We successfully performed manual tests with RATs based on HTTP, Telnet, and proprietary protocols by repeating the above test procedure. Using the sniffers of Figure 7, we captured all RAT traffic generated during this manual test stage and used it in forthcoming tests. Evidently, the large number of commands available in the repertoires of $R A T s$ renders manual testing very tedious and time-consuming.

As every individual $R A T$ command performs a well-defined operation and there is rarely dependency between different commands, a RAT server may not need to be "aware" of the sequence of client-issued commands and it is essentially memory-less. We exploit this memory-less characteristic to automatically generate test cases as follows: for each RAT under test, a session template ( $T C P$ or $U D P$ ) is first selected, then the template is filled with a sequence of appropriate command/response messages ${ }^{* *}$. The ensued traffic is injected into our RAT Catcher via a home-made IPS testing system [8] termed tester as shown in Figure 8. The tester can be configured to manipulate the traffic before it is replayed to our RAT Catcher, this manipulation includes modification of protocol fields such as $I P$ addresses, ports, checksums, and sequence numbers, replacement of packet payloads with arbitrary data, and/or re-shuffling packet orders.

For a $T C P$-based $R A T$ connection, the session template consists of three parts: connection establishment, message exchange, and connection termination. The first part contains the three-way handshake procedure with three packets, client-initiated SYN, $S Y N \mid A C K$ from the server, and client's confirmation ACK. The message exchange part

** based on the command set supported by individual RATs. 
contains a series of "command/response $(c / r)$ " pairs while the termination part is made up of two "FIN,ACK" pairs - one originated from the client and the second from the server. Clearly, packets in both connection establishment and termination have no payload and are fixed; packets in the message exchange part were generated automatically with the help of command sets and possible responses for the RAT under test. The UDP session template simply consists of a sequence of "command/response $(c / r)$ " pairs specified by testers or automatically generated. Such sequences of commands are randomly chosen from the command sets for the RATs under testing. In addition, the number of RAT sessions to be created and the appearance frequency of each command in the generated traffic are configurable. To simulate multiple concurrent sessions, our tester can generate an arbitrary number of test cases, interleave them together, and shuffle the replay order before feeding into the RAT Catcher.

We employed SubSeven in the automated testing stage to show the detection accuracy of both RAT Catcher and Snort. Overall, we generated 100,000 of TCP-based SubSeven sessions; each session contained a random number -in the range of $[1,20]-$ of "command/reply $(c / r)$ " pairs whose sizes and payloads were randomized unless specific format/parameter requirements are necessitated by the protocol. The generated RAT sessions were injected into both RAT Catcher and Snort. The outcome of the experiment appears in Table X. Sessions are grouped according to the number of their "command/reply $(c / r)$ " pairs and column cnt shows the number of Trojan-sessions per group. Column noreply shows the number of sessions from each group that do not trigger any server-reply. Our RAT Catcher initially uses one or more messages of a session to determine the potential RAT type of the session which subsequently is confirmed by additional messages received. The two columns RAT Catcher detect and RAT Catcher confirm indicate the number of sessions tentatively labeled as Trojan and those confirmed as such by our RAT Catcher. Table X also shows respective results obtained with Snort(v.2.2) having all specific signatures for detecting SubSeven enabled.

\begin{tabular}{|r|c|c|c|c|c|c|c|}
\hline "c/r" & cnt & no-reply & reply & RAT Catcher detect (\%) & RAT Catcher confirm (\%) & Snort detect (\%) & Snort miss (\%) \\
\hline 1 & 4865 & 118 & 4747 & $4865(100.00)$ & $4747(97.57)$ & $95(1.95)$ & $4770(98.05)$ \\
2 & 5062 & 2 & 5060 & $5062(100.00)$ & $5062(100.00)$ & $176(3.48)$ & $4886(96.52)$ \\
3 & 5069 & 0 & 5069 & $5069(100.00)$ & $5069(100.00)$ & $276(5.44)$ & $4793(94.56)$ \\
4 & 5318 & 0 & 5318 & $5318(100.00)$ & $5318(100.00)$ & $349(6.56)$ & $4969(93.44)$ \\
5 & 4911 & 0 & 4911 & $4911(100.00)$ & $4911(100.00)$ & $410(0.083)$ & $4501(91.65)$ \\
6 & 5171 & 0 & 5171 & $5171(100.00)$ & $5171(100.00)$ & $556(10.75)$ & $4615(89.25)$ \\
7 & 4979 & 0 & 4979 & $4979(100.00)$ & $4979(100.00)$ & $570(11.45)$ & $4409(88.55)$ \\
8 & 5306 & 0 & 5306 & $5306(100.00)$ & $5306(100.00)$ & $683(12.87)$ & $4623(87.13)$ \\
9 & 5038 & 0 & 5038 & $5038(100.00)$ & $5038(100.00)$ & $761(15.11)$ & $4277(84.89)$ \\
10 & 4857 & 0 & 4857 & $4857(100.00)$ & $4857(100.00)$ & $723(14.89)$ & $4134(85.11)$ \\
20 & 4879 & 0 & 4879 & $4879(100.00)$ & $4879(100.00)$ & $1503(30.81)$ & $3376(69.19)$ \\
\hline
\end{tabular}

Table X. Testing RAT Catcher and Snort using SubSeven-based test cases

Table X shows that the RAT Catcher detects all SubSeven sessions and its overall confirm rate is nearly perfect. Only in the first group, a few instances of unconfirmed Trojan connections appear as single message-sessions offering no option for confirmation. Nevertheless, all SubSeven are properly tagged and only $2.43 \%$ of $R A T$-sessions cannot be confirmed. On the contrary, the accuracy of Snort is far from satisfactory with worst $1.95 \%$ detection rate for group 1, best $30.81 \%$ for group 20, and average $16.59 \%$ for all cases. This is attributed to the fact the Snort uses only three rules for SubSeven. Clearly, we could craft signatures in Snort to cover all SubSeven commands but this would greatly burden its operation and deteriorate its performance to an unacceptable level. We repeated our testing for traffic generated by FTP, HTTP, and Telnet-based Trojans using synthetic background noise with the layout of Figure 8. We experimented with various intensities of background noise traffic regulated by the machines making up the noise generator and established very similar results to those shown in Table X.

In the last stage of our baseline experimentation, we dealt with Trojans that use encryption algorithms. As such RATs have extra dimensions of freedom-encryption algorithms and encryption keys-and share little commonality in their encryption processes, 


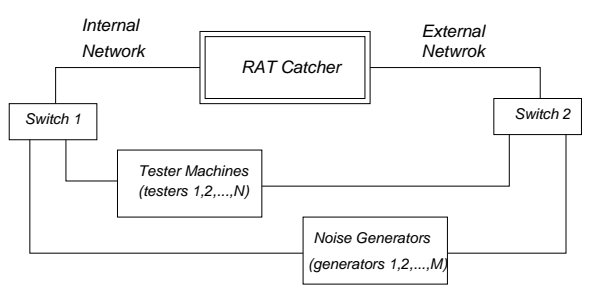

Figure 9. Testbed with multiple testers to generate RAT traffic and multiple testers for background noise

we designed special testing procedures that we discuss with the help of Back Orifice (BO)and NetBus Pro-generated traffic. We used the Internet available source-code for the BOclient and changed its encryption seed for each newly created session. The various streams of ensued traffic -resembling that of Table III- were fed into both RAT Catcher and Snort. In all instances, RAT Catcher and Snort maintain the same detection accuracy as both resort to application-layer protocol dissection. While experimenting with NetBus Pro, we deployed a synthetic approach as NetBus Pro uses a proprietary encryption algorithm and its source code is not known. We generated the 10-byte application protocol header and randomly selected payload for every created NetBus Pro packet. We generated upto 1,000,000 NetBus Pro sessions and injected this synthetic traffic to both RAT Catcher and Snort. Our experiments showed that the RAT Catcher creates no false negatives achieving the perfect detection rate while Snort fails to recognize most of the generated sessions. Snort appears to be ineffective as it can detect only those NetBus Pro sessions that happen to have client-initiated and server-originated packets with sizes of $0 \times 20$ and $0 \times 10$ Bytes, respectively. In contrast, the NetBus Pro protocol analyzer ${ }^{\dagger \dagger}$ of $R A T$ Catcher treats the message-size field as a variable and therefore can recognize NetBus Pro messages of any size. Moreover, the RAT Catcher resorts to message structure analysis and correlation of bi-directional traffic within a session to further improve its detection accuracy. We have repeated the same approach in experimenting with combinations of $R A T$ and attack-free traffic flows and obtained similar results for the performance of RAT Catcher and Snort. In summary, for Trojans based on HTTP, FTP, Telnet, or proprietary protocols, the RAT Catcher creates no false positives/negatives.

\subsection{Scalability and Performance Under Various Workloads}

As most RAT-clients are human-operated, the time gap between two consecutive commands submitted to the server is often long reflecting the attacker's thinking time. The time-stamped SubSeven message exchanges of Table VII indicate that this thinking time can be as long as 60 seconds -between messages 13 and 14-while on average is 40 seconds. This interactive nature of Trojans seems to indicate that processing overheads incurred by our RAT Catcher should not be a concern. However, as FortiGate is to be typically deployed at the edge between internal and external networks, it may often encounter in excess of half a million concurrent sessions and response times of corresponding sessions may be noticeably affected.

We evaluate the capabilities of the RAT Catcher under the above circumstances with the help of the testbed of Figure 9 and the traffic of Table IV. We use the latter as a template to generate various test cases and we split it into two parts: the first consists of the packets making up the normal TCP three-way handshake -not shown in the Table for brevity-and

${ }^{\dagger}{ }^{\dagger}$ of Algorithm 4 
packet 1; the second part contains the remaining packets $2-7$ as well as the four packets of the normal disconnection (also not shown). We configure our system to generate two alerts for each NetBus Pro session: the first is created when the session is marked as NetBus Pro by using data stream from the client to server and the second is raised when the session is confirmed as a true NetBus Pro with the help of data stream from the server to client. RAT Catcher generates the first alert when packet 1 is encountered and subsequently confirms the session after it has processed packet 2 . In our experimentation, we configure the RAT Catcher to forward all traffic even if Trojans have been detected and in our RAT Catcher implementation, we use the $L R U$ to replace sessions when the memory is full. As the generation of the second alert depends on the presence of the first alert and the availability of the session information, RAT Catcher may fail to raise this second alert if the RAT session in question is evicted due to memory congestion.

In all the tests performed in this section, $N=20$ machines are used as testers to create foreground traffic as follows: they replay the first half of the trace in Table IV for $n$ times, therefore generating $n$ RAT sessions; then pause for 1 second and then replay the second part of the trace for $n$ times; $n$ assumes values in the range of $[10,000,700,000]$. Meanwhile, we use $M=2$ noise-generator machines whose purpose is to create $m$ sessions of background traffic using the attack-free $W U-F T P D$ application $-m$ takes values in the range $[1,50,000]$. We also elect to introduce artificial delays in the replay of background traffic by splitting each $W U$-FTPD session into two parts and stall the noise-generators for 1 second in between replaying these two parts. Each time a trace is replayed, the testers modify $I P$ s and port numbers of both source and destination of connections to avoid conflicts among different replayed sessions. Similarly, the noise-generators also change protocol fields accordingly so that source and destination IPs and ports do not present conflicts. To simplify the coordination among different test machines, we assign nonoverlapping IP address ranges to different machines. For every test, we observe the behavior of RAT Catcher by recording the number of sessions that are correctly marked as NetBus Pro with both alerts generated, and calculate the ratio of such correctly marked sessions over the total $n$ replayed NetBus Pro sessions.

\begin{tabular}{|c|l|c|c|c|c|c|c|c|}
\hline case \# & $\downarrow m ;$ FTP delay & $n=10,000$ & $n=50,000$ & $n=75,000$ & $n=100,000$ & $n=200,000$ & $n=500,000$ & $n=700,000$ \\
\hline 1 & $1 ;$ no & 100.00 & 100.00 & 100.00 & 100.00 & 100.00 & 100.00 & 100.00 \\
\hline 2 & $1 ; 1$ second & 100.00 & 100.00 & 100.00 & 100.00 & 100.00 & 100.00 & 100.00 \\
\hline 3 & 10,$000 ;$ no & 100.00 & 100.00 & 100.00 & 100.00 & 100.00 & 100.00 & 100.00 \\
\hline 4 & 10,$000 ; 1$ second & 100.00 & 100.00 & 100.00 & 100.00 & 100.00 & 100.00 & 99.99 \\
\hline 5 & 20,$000 ;$ no & 100.00 & 100.00 & 100.00 & 100.00 & 100.00 & 100.00 & 100.00 \\
\hline 6 & 20,$000 ; 1$ second & 100.00 & 100.00 & 100.00 & 100.00 & 100.00 & 100.00 & 99.99 \\
\hline 7 & 30,$000 ;$ no & 100.00 & 100.00 & 100.00 & 100.00 & 100.00 & 100.00 & 100.00 \\
\hline 8 & 30,$000 ; 1$ second & 100.00 & 100.00 & 100.00 & 100.00 & 100.00 & 100.00 & 99.98 \\
\hline 9 & 40,$000 ;$ no & 100.00 & 100.00 & 100.00 & 100.00 & 100.00 & 100.00 & 100.00 \\
\hline 10 & 40,$000 ; 1$ second & 100.00 & 100.00 & 100.00 & 100.00 & 100.00 & 100.00 & 99.98 \\
\hline 11 & 50,$000 ;$ no & 100.00 & 100.00 & 100.00 & 100.00 & 100.00 & 100.00 & 100.00 \\
\hline 12 & 50,$000 ; 1$ second & 100.00 & 100.00 & 100.00 & 100.00 & 100.00 & 100.00 & 99.80 \\
\hline
\end{tabular}

Table XI. Test results of our RAT Catcher under various traffic workloads

Table XI shows the results of all our tests conducted under diverse workloads. The RAT Catcher demonstrates the correct behavior when no artificial delays are introduced by the noise-generators regardless of the volume of RAT traffic and intensity of the background noise. When artificial delays are in place while replaying WU-FTPD packets, the RAT Catcher fails to generate the second required alert -confirmation marking- for a small number of Trojan sessions as we increase the volumes of foreground and background traffic. Nevertheless, even for this small fraction of sessions, RAT Catcher still carries out the tentative marking. Overall, there is no RAT session completely missed by our framework. In addition, we also manually verify that no background traffic is mistakenly identified as RATs or dropped due to RAT Catcher malfunction. In all cases, the latency for SubSeven traffic is statistically the same with and without RAT Catcher present in FortiGate. By repeating the aforementioned procedure with other RATs including SubSeven, DeepThroat, BO2K, and NetSphere we obtain similar results. 


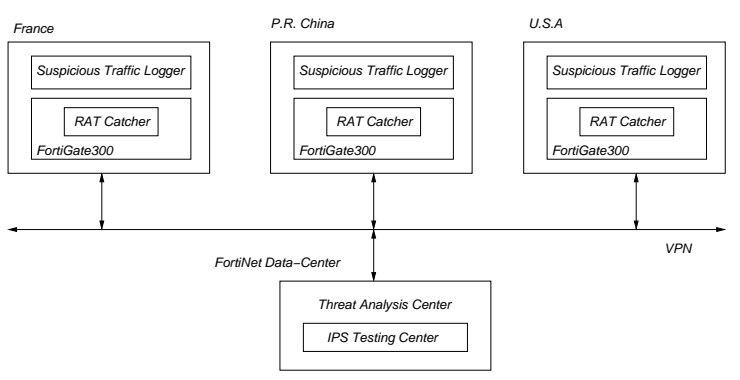

Figure 10. Deployment of RAT Catcher in France, P.R. China and United States

\subsection{The RAT Catcher in the Real World}

To evaluate the effectiveness of our approach in a real world setting, we have deployed FortiGate-300 devices in three higher education institutions in France, P.R. of China and the U.S.A. In collecting network-traffic data, we used the layout of Figure 10 to store and forward both confirmed and suspicious traffic streams containing attacks to a corporate Threat Analysis Center (TAC) for manual processing and verification purposes. In order to help discover new types of attacks and better ascertain the false-negative rate of FortiGate300, we augment RAT Catcher with the Suspicious Traffic Logger (STL) module that simply logs likely malicious yet not-locally resolved streams/sessions. Regional devices periodically transfer data on detected RATs and un-identified but suspect connections to $T A C$ where sampling and analyses on the traffic are performed. In a similar fashion, the collected suspicious traffic by STL is manually analyzed for new strains of Trojans. Once the confirmed Trojan traffic is stored, we also use Snort to assess its effectiveness by computing the false negative rate while portions of confirmed attack-free traffic from STL is replayed to Snort for gauging its false positive rate. From the forwarded traffic to TAC, we present all detected RAT instances for Back Orifice, NetBus and SubSeven during the period of June 26th, 2006 to July 9th, 2006 in Table XII.

\begin{tabular}{|l|l|l|l|l|l|l|l|l|l|}
\hline day & \multicolumn{3}{|c|}{ Back Orifice } & \multicolumn{3}{c|}{ NetBus } & \multicolumn{3}{c|}{ SubSeven } \\
\hline & US,CN,FR/Total & Catcher & Snort & US,CN,FR/Total & Catcher & Snort & US,CN,FR/Total & Catcher & Snort \\
\hline 1 & $204,115,46 / 365$ & $365 / 0$ & $365 / 0$ & $55,25,17 / 97$ & $97 / 0$ & $63 / 34$ & $32,15,4 / 51$ & $51 / 0$ & $33 / 18$ \\
2 & $87,75,29 / 191$ & $191 / 0$ & $191 / 0$ & $86,68,36 / 190$ & $190 / 0$ & $143 / 47$ & $26,12,16 / 54$ & $54 / 0$ & $32 / 22$ \\
3 & $197,132,104 / 433$ & $433 / 0$ & $433 / 0$ & $53,32,10 / 95$ & $95 / 0$ & $64 / 31$ & $11,6,8 / 25$ & $25 / 0$ & $16 / 9$ \\
4 & $168,64,40 / 272$ & $272 / 0$ & $272 / 0$ & $108,47,32 / 187$ & $187 / 0$ & $117 / 70$ & $46,34,11 / 91$ & $91 / 0$ & $62 / 29$ \\
5 & $265,119,14 / 398$ & $398 / 0$ & $398 / 0$ & $56,34,10 / 100$ & $100 / 0$ & $74 / 26$ & $36,24,2 / 62$ & $62 / 0$ & $36 / 26$ \\
6 & $96,51,67 / 214$ & $214 / 0$ & $214 / 0$ & $89,36,4 / 129$ & $129 / 0$ & $84 / 45$ & $17,8,2 / 27$ & $27 / 0$ & $19 / 8$ \\
7 & $230,95,14 / 339$ & $339 / 0$ & $339 / 0$ & $51,28,17 / 96$ & $96 / 0$ & $47 / 49$ & $33,25,7 / 65$ & $65 / 0$ & $49 / 16$ \\
8 & $205,121,99 / 425$ & $425 / 0$ & $425 / 0$ & $115,50,29 / 194$ & $194 / 0$ & $90 / 104$ & $50,26,15 / 91$ & $91 / 0$ & $59 / 32$ \\
9 & $43,25,17 / 85$ & $85 / 0$ & $85 / 0$ & $65,41,16 / 122$ & $122 / 0$ & $56 / 66$ & $12,4,3 / 19$ & $19 / 0$ & $15 / 4$ \\
10 & $218,107,18 / 343$ & $343 / 0$ & $343 / 0$ & $66,29,11 / 106$ & $106 / 0$ & $77 / 29$ & $28,20,14 / 62$ & $62 / 0$ & $27 / 35$ \\
11 & $125,64,37 / 226$ & $226 / 0$ & $226 / 0$ & $79,53,32 / 164$ & $164 / 0$ & $109 / 55$ & $42,16,3 / 61$ & $61 / 0$ & $37 / 24$ \\
12 & $182,93,43 / 318$ & $318 / 0$ & $318 / 0$ & $77,30,19 / 126$ & $126 / 0$ & $67 / 59$ & $42,18,19 / 79$ & $79 / 0$ & $45 / 34$ \\
13 & $246,123,51 / 420$ & $420 / 0$ & $420 / 0$ & $104,53,24 / 181$ & $181 / 0$ & $122 / 59$ & $49,27,13 / 89$ & $89 / 0$ & $38 / 51$ \\
14 & $96,54,13 / 163$ & $163 / 0$ & $163 / 0$ & $56,31,30 / 117$ & $117 / 0$ & $80 / 37$ & $38,17,17 / 72$ & $72 / 0$ & $40 / 32$ \\
\hline
\end{tabular}

Table XII. Detection rates by RAT Catcher and Snort in our real-world traffic

Table XII points out most RAT instances occur in U.S. followed by China and France; Back Orifice is the most frequently found $R A T$ in the three regions, followed by NetBus and SubSeven as shown in column US,CN,FR/Total. In TAC, we manually establish that all RAT sessions shown in Table XII detected and reported by our regionally placed RAT Catchers have been indeed correctly identified.

Table XII shows the detection/prevention results and false negatives by RAT Catcher and Snort in Columns Catcher and Snort in the format of correct/error, should all identified $R A T$ sessions at TAC be replayed to RAT Catcher and Snort when both are configured to 
block all RAT sessions. Clearly, RAT Catcher does not create any false positives/negatives and successfully blocks all identified RAT connections. Moreover, it typically determines the application type of a session after inspecting the first message from each direction of the session; so, it effectively forwards no attacker-submitted command and minimizes potential damage to victims. On the other hand, Snort detects all Back Orifice connections as it integrates a dedicated protocol dissector which performance-wise is equivalent to that of our RAT Catcher. However, Snort misses a number of NetBus and SubSeven connections, generating false negatives since the injected traffic has been manually evaluated to be malicious. For instance, Snort rule 3009 [9] used to identify NetBus Pro contains pattern " $B N|20000200|$ " where the byte sequence "|20 $00 \mid$ " immediately following string " $B N$ " designates the size of the message. Obviously, Snort rule 3009 assumes that all NetBus Pro messages with command-code $0 \times 05$ should be $0 \times 20$ bytes. However, the sizes of NetBus Pro messages with command-code 0x05 can vary in different sessions as demonstrated by the captured traffic shown in the first part of Table XIII; this leads to a false negative by the Snort rules in place as the connection request from the NetBus Pro-client has size of 31 bytes $(0 x l F)$ instead of 32 bytes $(0 \times 20)$.

\begin{tabular}{|c|c|c|c|}
\hline \# & dir & payload & description \\
\hline \multicolumn{4}{|c|}{ NetBuS Pro traffic - protocol: $T C P$; attacker denoted as A; victim denoted as $\mathrm{V}$} \\
\hline 1 & $\mathrm{~A}: 35821->\mathrm{V}: 20034$ & $\begin{array}{l}B N \mid 1 \mathrm{~F} 000200 \text { DC } 330500410 \mathrm{C} 691 \mathrm{~F} \mid \\
\mid 5 \mathrm{D} 28 \text { 5B } 95 \text { 9C AD } 95 \text { A8 E6 28 FD... }\end{array}$ & $\begin{array}{l}\text { msg starts with } B N \text {; size: } 0 x 1 \mathrm{~F} ; \text { ver: } \\
\text { 0x02; cmd: } 0 \mathrm{x} 05 \text { (connection establishment); }\end{array}$ \\
\hline 2 & $\mathrm{~V}: 20034->\mathrm{A}: 35821$ & $B N|10000200 \mathrm{DC} 330500410 \mathrm{C} 691 \mathrm{~F} 5 \mathrm{D} 28|$ & reply msg; size: 0x10; ver: 0x02; cmd: 0x05 (con. est.); \\
\hline \multicolumn{4}{|c|}{ Normal $F T P$-traffic - protocol: $T C P ; F T P$ server as $\mathrm{S} ;$ FTP client denoted as $\mathrm{C}$} \\
\hline 1 & $\mathrm{C}: 46943->\mathrm{S}: 21$ & PASV|0D 0A| & client requests "passive" mode \\
\hline 2 & $\mathrm{~S}: 21->\mathrm{C}: 46943$ & 227 Entering Passive Mode (x, y, z, w, 66, 63) & server listens on port $16959(66 * 256+63)$ \\
\hline 3 & C:46944->S:16959 & $(\mathrm{SYN})$ & client requests data connection \\
\hline 4 & S:16959- >C:46944 & (SYN|ACK) & server accepts the data connection \\
\hline 5 & C:46944->S:16959 & (ACK) & client confirms data connection \\
\hline 6 & C:46943- >S:21 & RETR commands.txt $|0 \mathrm{D} 0 \mathrm{~A}|$ & client requests file "commands.txt" \\
\hline 7 & $S: 21->C: 46943$ & 150 ASCII data connection for commands.txt & response from server \\
\hline 8 & S:16959- >C:46944 & PWD - print name of current/working directory & content of file "commands.txt" \\
\hline
\end{tabular}

Table XIII. Traffic causing Snort to generate false positives and negatives

By replaying STL-collected traffic that has been manually verified as legitimate, we can establish that Snort also generates false-positives. For example, the second part of Table XIII shows a normal FTP session and its data connection for a file transfer. In message 1, the FTP-client requests "passive mode" which is approved by the server in message 2. At the same time, the server also informs the client of its intent to use the port 16959 for the data connection. In messages 3 to 5, a new TCP connection is established and subsequently used to deliver the content of the file commands.txt requested by the client in message 6. As the pattern $P W D$ is part of the transported content of message 8 , it triggers Snort rule 107 discussed in [9] which is obviously a false alarm. Overall, we have observed that RAT Catcher correctly identifies and subsequently blocks traffic streams known to be the result of RATs whereas Snort lags behind due to limited number of specific rules for each Trojan, yielding both false positives and negatives.

\section{Conclusions and Future Work}

A Remote Administration Trojan (RAT) is a malicious program that allows an attacker to remotely control a computing system often creating irrevocable damage. Existing techniques including fingerprinting, auto-start monitoring, surveillance of network activities and packet analysis using static signatures and/or fixed communication ports are limited in both scope and effectiveness. Today, traffic obfuscation, port hopping, file renaming and compression, information encryption along with evasion techniques work counter to the effectiveness and efficiency of anti-Trojan systems. In this paper, we propose the RAT Catcher, a network-based framework for Trojan detection that operates in inline fashion at the edge of the network and reliably identifies $R A T$ activities. 
The RAT Catcher inspects every passing packet and maintains information for the entire lifetime of sessions created by both Trojans and normal applications. This session tracking improves detection accuracy by providing stateful inspection as well as intra-session and inter-session data correlation. The RAT Catcher stores all packets in every data stream, reassembles them, and interprets the resulting data aggregations according to known Trojan protocols. To this end, the framework performs deep inspection on data streams by scanning protocol fields and whenever feasible message content. The RAT Catcher dissects both data streams within a session and correlates them in order to ensure that the traffic in both directions complies with protocol specifications defined by RAT systems in terms of syntax and semantics. By analyzing the syntax/format of application messages and inspecting the exchange order of messages between clients and servers, our RAT Catcher can defeat evasion techniques. By associating RAT control and data channels, correlating active with defunct sessions, and restoring boundaries of application messages through reassembly, the RAT Catcher does not generate false positives or negatives. Actions imposed on identified Trojan sessions include alerting, packet blocking, session take-over, and connection termination.

Experiments showed that the proposed framework is both effective and efficient. Subjected to comprehensive testing, the RAT Catcher demonstrated wide RAT coverage, excellent detection accuracy, and low processing overheads. We plan to further pursue our work in the area in at least three directions: first, we will keep enhancing our RAT Catcher so that it can deal with new types of Trojans as the latter become known; second, develop advanced techniques to identify RATs that use strong cryptographic mechanisms and finally, explore the integration of our RAT Catcher with other security systems including firewalls, anti-virus, and anti-malware programs to more effectively combat aggregate malicious activities resulting from the mixture of RAT and popular worms known as

\section{Blended Threats.}

Acknowledgments: We are very grateful to the anonymous reviewers for their detailed comments and suggestions that helped us significantly improve our work. We also thank Qinghong Yi, Gary Duan, Ping Wu, Joe Zhu, Yelin Guan, and Hong Huang for helping us with various aspects of the RAT Catcher implementation and testing and Prof. Boris Aronov for comments on earlier versions of the manuscript.

\section{REFERENCES}

1. C. M. Adams and S. E. Tavaris. Designing S-Boxes for Ciphers Resistant To Differential Cryptanalysis. In Proceedings of the 3rd Symposium on State and Progress of Research in Cryptography, pages 181-190, Rome, Italy, Feb. 1993.

2. L. Adelman. An Abstract Theory of Computer Viruses. In Proceedings of Advances in Cryptology (CRYPTO'88), pages 354-374, New York, NY, Aug. 1988. Springer-Verlag.

3. Korea Information Security Agency. Security Incident Statistics in Korea. http://www.kisa.or.kr/english/statistics/hack/, 2000.

4. D. Bell and L. LaPadula. Secure Computer Systems: Unified Exposition and MULTICS Interpretation. Technical report, MITRE Corporation, Bedford, MA, July 1975. MTR-2997.

5. M. Bishop. A Model of Security Monitoring. In Proceedings of the Fifth Annual Computer Security Applications Conference, pages 46-52, Dec. 1989.

6. CERT. Advisory CA-1999-01: Trojan Horse Version of TCP Wrappers. http://www.cert.org/advisories/CA1999-01.html, 1999.

7. CERT. Advisory CA-1999-02: Trojan Horses. http://www.cert.org/advisories/CA-1999-02.html, 1999.

8. Z. Chen, P. Wei, and A. Delis. A Pragmatic Methodology for Testing Intrusion Prevention Systems (IPSs). Technical report, Deprt. of Informatics \& Telecommunications, Univ. of Athens, Athens, Greece, August 2004.

9. Z. Chen, P. Wei, and A. Delis. Catching Remote Administration Trojans. Technical report, Deprt. of Informatics \& Telecommunications, Univ. of Athens, Athens, Greece, May 2007. www.di.uoa.gr/ ad.

10. D. Clark and D. Wilson. A Comparison of Commercial and Military Computer Security Policies. In Proceedings of the 1987 Symposium on Security and Privacy, pages 184-194, Apr. 1987.

11. F. Cohen. Computer Viruses: Theory and Experiments. Computers and Security, 6(1):22-35, Feb. 1987.

12. F. Cohen. Computational Aspects of Computer Viruses. Computers and Security, 8(4):325-344, Jun. 1989.

13. F. Cohen. Practical Defenses Against Computer Viruses. Computers and Security, 8(2):149-160, Apr. 1989.

14. Symantec Com. Norton Anti-Virus System. http://www.symantec.com/.

15. D. E. Comer. Internetworking with TCP/IP: Principles, Protocols, and Architecture. Prentice-Hall, Englewood Cliffs, NJ, 1991. 
16. Commodon. Threats to Your Security on the Internet-SubSeven. http://www.commodon.com, 2001.

17. T. H. Cormen, C. E. Leiserson, and R. L. Rivest. Introduction to Algorithms. The MIT Press, Boston, MA, 1997.

18. Privacy Software Corporation. Anti-Trojan Program: BOClean. http://www.nsclean.com/trolist.html, 2004.

19. H. DeMaio. Viruses - Management Issue. Computers and Security, 8(5):381-388, Oct. 1989.

20. D. Denning. An Intrusion-Detection Model. IEEE Transactions on Software Engineering, SE-13(2):222232, Feb. 1987.

21. D. Denning. The Science of Computing: Computer Viruses. American Scientist, 76(3):236-238, May 1988.

22. T. Duff. Experiences with Viruses on UNIX Systems. Computing Systems, 2(2):155-172, Spring 1989.

23. Ethereal. Ethereal: Powerful Multi-Platform Analysis. http://www.ethereal.com, May 2004.

24. R. Farrow. UNIX System Security. Addison-Wesley Publishing Co., Reading, MA, 1991.

25. R. Fielding, J. Gettys, J. Mogul, H. Frystyk, L. Masinter, P. Leach, and T. B. Lee. Hypertext Transfer Protocol - HTTP/1.1. Internet Engineering Task Force, June 1999.

26. S. Garfinkel and G. Spafford. Practical UNIX Security. O'Reilly and Associates, Sebastopol, CA, 1991.

27. W. Gleissner. A Mathematical Theory for the Spread of Computer Viruses. Computers and Security, 8(1):35-41, Feb. 1989.

28. S. Gordon and D. M. Chess. Attitude Adjustment: Trojans and Malware on the Internet. In Proceedings of the EICAR, pages 183-204, Copenhaguen, Denmark, May 1999.

29. R. Hansen. Moose Test for Windows: NetBus Pro and How it Happened. http://www.heise.de/ct/english/99/17/088/, 1999.

30. H. M. Heys and S. E. Tavares. On the Security of the CAST Encryption Algorithm. In Proc. of Canadian Conf. on El. \& Comp. Eng., Halifax, Canada, Sep. 1994.

31. L. Hoffman. Rogue Programs: Viruses, Worms, and Trojan Horses. Van Nostrand Reinhold, New York City, NY, 1990.

32. Fortinet Inc. Intrusion Prevention System. Web Site: www.fortinet.com, Oct. 2004.

33. Foundstone Inc. FPort: Intrusion Detection Tool. http://www.foundstone.com/.

34. M. Joseph and A. Avizienis. A Fault Tolerant Approach to Computer Viruses. In Proceedings of the 1988 Symposium on Security and Privacy, pages 52-58, Oakland, CA, Apr. 1988.

35. G. H. Kim and E. H. Spafford. The Design and Implementation of Tripwire: A File System Integrity Checker. In ACM Conference on Computer and Communication Security, pages 18-29, Fairfax, VA, 1994.

36. S. Kulakow. NetBus 2.1: Is It Still a Trojan Horse or an Actual Valid Remote Control Administration Tool? Web page, 2001.

37. S. Lipner. Non-Discretionary Controls for Commercial Applications. In Proceedings of the 1982 Symposium on Security and Privacy, pages 2-10, Apr. 1982.

38. Zone Labs LLC. ZoneAlarm Security Suite. http://www.zonelabs.com/, 2004.

39. T. Lunt and R. Jagannathan. A Prototype Real-Time Intrusion-Detection Expert System. In Proceedings of the 1988 Symposium on Security and Privacy, pages 59-66, Apr. 1988.

40. PC Magazine. StartupCop Pro. http://www.pcmag.com/.

41. H. Nussbacker. Israeli Internet Hacking Analysis for 2000. In Proceedings of the Internet Society of Israel Conference, Tel Aviv, Israel, March 2001. http://www.isoc.org.il/conf2001/presentations/nussbackerl.ppt.

42. The Cult of the Dead Cow. Back Orifice 2000. http://www.bo2k.com or http://www.cultdeadcow.com/, 2004.

43. PestPatrol. About RATS: SubSeven and Remote Administration Trojans. http://www.pestpatrol.com/ whiterpapers, 2006

44. M. Roesch. Snort - Lightweight Intrusion Detection for Networks. In USENIX 13-th Systems Administration Conference - LISA'99, Seattle, Washingto, 1999.

45. J. Saltzer and M. Schroeder. The Protection of Information in Computer Systems. Proceedings of the IEEE, 63(9):1278-1308, Sep. 1975.

46. B. Schneier. Applied Cryptography, Protocols, Algorithms, and Source Code in C, Second Edition. John Wiley \& Sons, Inc., 1996.

47. Mischel Internet Security. TrojanHunter vs. the Parasitic Beast Trojan. http://www.misec.net/papers/thvsbeast/, 2004.

48. D. D. Sleator and R. E. Tarjan. Self-Adjusting Binary Search Trees. Journal of the ACM, 32(3):652-686, 1985.

49. Winternals Software. TCPView Pro. http://www.winternals.com/.

50. D. Song, G. Shaffer, and M. Undy. Nidsbench - A Network Intrusion Detection Test Suite. In Second International Workshop on Recent Advances in Intrusion Detection (RAID 1999), West Lafayette, 1999.

51. Inc. Sourcefire. Snort 2.0: Detection Revisited. White Paper, February 2003.

52. E. Spafford. The Internet Worm Program: An Analysis. ACM Computer Communications Review, 19(1), Jan. 1989.

53. R. C. Summers. Secure Computing Threats and Safeguards. McGraw-Hill, 1997.

54. M. Swimmer. Dynamic Detection and Classification of Computer Viruses. In Proceedings of the Sixth International Virus Bulletin Conference, pages 149-159. Virus Bulletin Ltd, 1996.

55. Internet Security Systems. ISS Security Alert: Windows Backdoor Update III. http://www.xforce.iss.net, 1999.

56. H. Teng, K. Chen, and S. Lu. Adaptive Real-Time Anomaly Detection Using Inductively Generated Sequential Patterns. In Proceedings of the 1990 Symposium on Research in Security and Privacy, pages 278-284, May 1990.

57. I. Whalley. Testing Times for Trojans. In Proceedings of the Ninth International Virus Bulletin Conference, pages 55-67, September/October 1999.

58. C. Young. Taxonomy of Computer Virus Defense Mechanisms. In Proceedings of the Tenth National Computer Security Conference, pages 220-225, Oakland, CA, Sep. 1987. 